\title{
MOA-2011-BLG-262Lb: A SUB-EARTH-MASS MOON ORBITING A GAS GIANT PRIMARY OR A HIGH VELOCITY PLANETARY SYSTEM IN THE GALACTIC BULGE
}

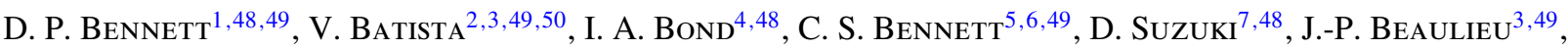 \\ A. Udalski ${ }^{8,51}$, J. DonatowiCz $^{9}$, V. BozzA ${ }^{10,11}$, \\ AND
}

F. Abe ${ }^{12}$, C. S. Botzler ${ }^{13}$, M. Freeman ${ }^{13}$, D. Fukunaga ${ }^{12}$, A. Fukui ${ }^{14}$, Y. Itow ${ }^{12}$, N. Koshimoto ${ }^{7}$, C. H. Ling ${ }^{4}$, K. Masuda ${ }^{12}$, Y. Matsubara ${ }^{12}$, Y. Muraki ${ }^{12}$, S. Namba ${ }^{7}$, K. Ohnishi ${ }^{15}$, N. J. Rattenbury ${ }^{13}$, To. Saito $^{16}$, D. J. Sullivan ${ }^{17}$, T. Sumi ${ }^{7}$, W. L. Sweatman ${ }^{4}$, P. J. Tristram ${ }^{18}$, N. Tsurumi ${ }^{12}$, K. Wada ${ }^{7}$, P. C. M. Yock ${ }^{13}$

(The MOA Collaboration)

M. D. Albrow ${ }^{19}$, E. Bachelet ${ }^{20}$, S. Brillant ${ }^{21}$, J. A. R. Caldwell ${ }^{22}$, A. Cassan ${ }^{3}$, A. A. Cole ${ }^{23}$, E. Corrales ${ }^{3}$, C. Coutures ${ }^{3}$, S. Dieters ${ }^{23}$, D. Dominis Prester ${ }^{24}$, P. Fouqué ${ }^{20}$, J. Greennill ${ }^{23}$, K. Horne ${ }^{25,52}$, J.-R. Koo ${ }^{26}$, D. Kubas ${ }^{3}$, J.-B. Marquette ${ }^{3}$, R. Martin ${ }^{27}$, J. W. Menzies ${ }^{28}$, K. C. Sahu $^{29}$, J. Wambsganss ${ }^{30}$, A. Williams ${ }^{27}$, M. Zub ${ }^{30}$

(The PLANET COLlaboration)

J. Y. $\mathrm{ChO}^{26}$, D. L. DePoy ${ }^{31}$, Subo Dong ${ }^{32}$, B. S. Gaudi ${ }^{2}$, A. Gould ${ }^{2}$, C. $\mathrm{HAN}^{26}$, C. B. Henderson ${ }^{2}$, D. McGregor ${ }^{2}$, C.-U. LeE ${ }^{33}$, R. W. PoGGE ${ }^{2}$, I.-G. Shin ${ }^{26}$, J. C. YeE ${ }^{2,53}$

(THE $\mu$ FUN COLLABORATION)

M. K. Szymański ${ }^{8}$, J. Skowron ${ }^{8}$, R. Poleski ${ }^{2,8}$, S. KozŁowski ${ }^{8}$, Ł. Wyrzykowski ${ }^{8}$,34, M. Kubiak ${ }^{8}$, P. Pietrukowicz ${ }^{8}$, G. PIETRZYŃSKI ${ }^{8,35}$, I. SOSZYŃSKI ${ }^{8}, \mathrm{~K}$ UlaCZYK $^{8}$

(The OGLE COLlaboration)

Y. Tsapras ${ }^{36,37}$, R. A. Street ${ }^{36}$, M. DominiK ${ }^{25,54}$, D. M. Bramich ${ }^{38,39}$, P. Browne ${ }^{25}$, M. Hundertmark ${ }^{25}$, N. Kains ${ }^{38}$, C. SNOdgrass ${ }^{40}$, I. A. Steele ${ }^{41}$

(The Robonet Collaboration)

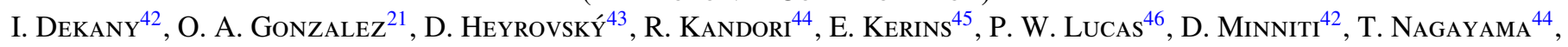
M. ReJKuba ${ }^{21}$, A. C. Robin ${ }^{47}$, And R. SAIto ${ }^{42}$

\footnotetext{
${ }^{1}$ Department of Physics, University of Notre Dame, 225 Nieuwland Science Hall, Notre Dame, IN 46556, USA; bennett@nd.edu

2 Department of Astronomy, Ohio State University, 140 West 18th Avenue, Columbus, OH 43210, USA

${ }^{3}$ UPMC-CNRS, UMR 7095, Institut d'Astrophysique de Paris, 98bis Boulevard Arago, F-75014 Paris, France

${ }^{4}$ Institute of Natural and Mathematical Sciences, Massey University, Auckland 0745, New Zealand

${ }^{5}$ Department of Physics, Massachussets Institute of Technology, Cambridge, MA 02139, USA

${ }^{6}$ Goddard Space Flight Center, Greenbelt, MD 20771, USA

${ }^{7}$ Department of Earth and Space Science, Osaka University, Osaka 560-0043, Japan

${ }^{8}$ Warsaw University Observatory, Al. Ujazdowskie 4, 00-478 Warszawa, Poland

${ }^{9}$ Technische Universität Wien, Wieder Hauptst. 8-10, A-1040 Vienna, Austria

${ }^{10}$ Dipartimento di Fisica, Università di Salerno, Via Ponte Don Melillo 132, I-84084 Fisciano, Italy

${ }^{11}$ Istituto Nazionale di Fisica Nucleare, Sezione di Napoli, Via Cintia, I-80126 Napoli, Italy

${ }^{12}$ Solar-Terrestrial Environment Laboratory, Nagoya University, Nagoya 464-8601, Japan

${ }^{13}$ Department of Physics, University of Auckland, Private Bag 92-019, Auckland 1001, New Zealand

${ }^{14}$ Okayama Astrophysical Observatory, National Astronomical Observatory of Japan, Okayama 719-0232, Japan

${ }_{16}^{15}$ Nagano National College of Technology, Nagano 381-8550, Japan

16 Tokyo Metropolitan College of Aeronautics, Tokyo 116-8523, Japan

${ }^{17}$ School of Chemical and Physical Sciences, Victoria University, Wellington 6140, New Zealand

${ }^{18}$ Mt. John University Observatory, P.O. Box 56, Lake Tekapo 8770, New Zealand

${ }^{19}$ Department of Physics and Astronomy, University of Canterbury, Christchurch 8020, New Zealand

${ }^{20}$ IRAP, CNRS, Université de Toulouse, F-31400 Toulouse, France

${ }^{21}$ European Southern Observatory, Casilla 19001, Vitacura 19, Santiago, Chile

${ }^{22}$ McDonald Observatory, Fort Davis, TX 79734, USA

${ }^{23}$ School of Mathematics and Physics, University of Tasmania, Hobart, TAS 7001, Australia

${ }^{24}$ Department of Physics, University of Rijeka, Omladinska 14, 51000 Rijeka, Croatia

${ }^{25}$ SUPA, University of St Andrews, School of Physics \& Astronomy, St Andrews KY16 9SS, UK

${ }^{26}$ Department of Physics, Chungbuk National University, Chongju 371-763, Korea

${ }_{27}$ Perth Observatory, Walnut Road, Bickley, Perth, WA 6076, Australia

${ }^{28}$ South African Astronomical Observatory, P.O. Box 9 Observatory 7925, South Africa

${ }^{29}$ Space Telescope Science Institute, 3700 San Martin Drive, Baltimore, MD 21218, USA

${ }^{30}$ Astronomisches Rechen-Institut, Zentrum für Astronomie der Universität Heidelberg (ZAH), D-69120 Heidelberg, Germany

${ }^{31}$ Department of Physics, Texas A\&M University, College Station, TX 77843-4242, USA

${ }^{32}$ Kavli Institute for Astronomy and Astrophysics, Peking University, Hai Dian District, Beijing 100871, China

${ }^{33}$ Korea Astronomy and Space Science Institute, Yuseong-gu 305-348 Daejeon, Korea

${ }^{34}$ Institute of Astronomy, University of Cambridge, Madingley Road, Cambridge CB3 OHA, UK

${ }^{35}$ Departamento de Astronomía, Universidad de Concepción, Casilla 160-C, Concepción, Chile

${ }^{36}$ Las Cumbres Observatory Global Telescope Network, 6740B Cortona Drive, Goleta, CA 93117, USA

${ }^{37}$ School of Physics and Astronomy, Queen Mary University of London, Mile End Road, London E1 4NS, UK

${ }^{38}$ ESO Headquarters, Karl-Schwarzschild-Str. 2, D-85748 Garching bei München, Germany

${ }^{39}$ Qatar Environment and Energy Research Institute, Qatar Foundation, Doha, Qatar

${ }^{40}$ Max Planck Institute for Solar System Research, D-37191 Katlenburg-Lindau, Germany

${ }^{41}$ Astrophysics Research Institute, Liverpool John Moores University, Liverpool CH41 1LD, UK

${ }^{42}$ Pontificia Universidad Catolica de Chile, Casilla 306, Santiago 22, Chile
} 


\author{
${ }^{43}$ Institute of Theoretical Physics, Charles University, 18000 Prague, Czech Republic \\ ${ }^{44}$ Graduate School of Science, Nagoya University, Furo-cho, Chikusa-ku, Nagoya 464-8602, Japan \\ 45 Jodrell Bank Centre for Astrophysics, University of Manchester, Oxford Road, Manchester M13 9PL, UK \\ ${ }^{46}$ University of Hertfordshire, Hatfield, Hertfordshire AL10 9AB, UK \\ ${ }^{47}$ Observatoire de Besancon Institut UTINAM, Universite Franche-Comte, CNRS-UMR 6213, BP 1615, F-25010 Besancon Cedex, France \\ Received 2013 December 14; accepted 2014 February 20; published 2014 April 7
}

\begin{abstract}
We present the first microlensing candidate for a free-floating exoplanet-exomoon system, MOA-2011-BLG-262, with a primary lens mass of $M_{\text {host }} \sim 4$ Jupiter masses hosting a sub-Earth mass moon. The argument for an exomoon hinges on the system being relatively close to the Sun. The data constrain the product $M_{L} \pi_{\text {rel }}$ where $M_{L}$ is the lens system mass and $\pi_{\text {rel }}$ is the lens-source relative parallax. If the lens system is nearby (large $\pi_{\text {rel }}$ ), then $M_{L}$ is small (a few Jupiter masses) and the companion is a sub-Earth-mass exomoon. The best-fit solution has a large

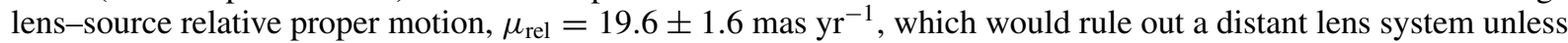
the source star has an unusually high proper motion. However, data from the OGLE collaboration nearly rule out a high source proper motion, so the exoplanet+exomoon model is the favored interpretation for the best fit model. However, there is an alternate solution that has a lower proper motion and fits the data almost as well. This solution is compatible with a distant (so stellar) host. A Bayesian analysis does not favor the exoplanet+exomoon interpretation, so Occam's razor favors a lens system in the bulge with host and companion masses of $M_{\text {host }}=0.12_{-0.06}^{+0.19} M_{\odot}$ and $m_{\text {comp }}=18_{-10}^{+28} M_{\oplus}$, at a projected separation of $a_{\perp}=0.84_{-0.14}^{+0.25} \mathrm{AU}$. The existence of this degeneracy is an unlucky accident, so current microlensing experiments are in principle sensitive to exomoons. In some circumstances, it will be possible to definitively establish the mass of such lens systems through the microlensing parallax effect. Future experiments will be sensitive to less extreme exomoons.
\end{abstract}

Key words: gravitational lensing: micro - planetary systems

Online-only material: color figure

\section{INTRODUCTION}

Gravitational microlensing occupies a unique niche among planet detection methods (Bennett 2008; Gaudi 2012). While the radial velocity (Butler et al. 2006; Mayor \& Queloz 2012) and transit methods (Borucki et al. 2011; Batalha et al. 2013) are most sensitive to planets in short period orbits, microlensing is most sensitive to planets orbiting beyond the snow line (Mao \& Paczyński 1991; Gould \& Loeb 1992) where the leading theory of planet formation, core accretion (Lissauer 1993; Pollack et al. 1996), predicts that the most massive planets should form. Ices, including water ice, can condense beyond the snow line (Ida \& Lin 2005; Lecar 2006; Kennedy et al. 2006; Kennedy \& Kenyon 2008; Thommes et al. 2008), and this means that the density of solid material in the proto-planetary disk increases by a factor of a few beyond the snow line, so that solid giant planet cores can form more rapidly. This is important because the hydrogen and helium that comprise the vast majority of the mass of gas giant planets is thought to be removed from proto-planetary disks in a few million years, so if gas giants do not form relatively quickly, they cannot form at all. For this reason, theory suggests that it could be difficult for gas giants to form around M-dwarfs (Laughlin et al. 2004).

Radial velocity observations (Johnson et al. 2007, 2010) seemed to confirm this picture, but microlensing observations (Gould et al. 2010; Cassan et al. 2012) paint a somewhat more complicated picture. Microlensing finds that $17_{-9}^{+6} \%$ of stars

\footnotetext{
${ }_{48}^{48}$ Microlensing Observations in Astrophysics (MOA) Collaboration.

${ }^{49}$ Probing Lensing Anomalies NETwork (PLANET) Collaboration.

${ }^{50}$ Microlensing Follow-up Network ( $\mu$ FUN Collaboration).

${ }^{51}$ Optical Gravitational Lensing Experiment (OGLE) Collaboration.

52 RoboNet Collaboration.

${ }^{53}$ Sagan Fellow; Harvard-Smithsonian Center for Astrophysics, 60 Garden Street, Cambridge, MA 02138, USA.

${ }^{54}$ Royal Society University Research Fellow.
}

have gas giant planets above 0.3 Jupiter masses (Cassan et al. 2012) with a host star sample strongly dominated by M-dwarfs. However, the planets found by microlensing generally orbit beyond the snow line, and the gas giants found by microlensing are often low-mass gas giants with a mass similar to Saturn (at 0.3 Jupiter masses; Gould et al. 2010). The combination of the relatively low-masses and wide orbits for the gas giants found by microlensing along with their relatively wide orbits means that analogs of most of the gas giant planets found by microlensing would not have been detected in the radial velocity surveys conducted to date. However, a recent combined analysis of precise radial velocity measurements and highcontrast imaging has been used to derive occurrence frequency of gas giants orbiting M-dwarfs beyond the snow line (Montet et al. 2014), and they find that their results are consistent with the microlensing results (Gould et al. 2010; Cassan et al. 2012).

Microlensing results also indicate that super-Earths or Neptunes are substantially more common than gas giants beyond the snow line (Sumi et al. 2010; Cassan et al. 2012). This is roughly in line with the predictions of the core accretion theory (Lissauer 1993; Pollack et al. 1996), which predicts that "failed Jupiters" with masses of the order of $\sim 10 M_{\oplus}$ should be quite common. These "failed Jupiters" are expected to be much more common than gas-giants for M-dwarf stellar hosts (Laughlin et al. 2004), although some have argued that the gravitational instability model could also make such planets (Boss 2006).

Perhaps the most surprising microlensing result to date was the discovery of a large population of planetary mass objects with no detectable host star (Sumi et al. 2011). While some of these could be bound planets in wide orbits (Quanz et al. 2012), the median separation of these planets is probably $>30 \mathrm{AU}$ (Bennett et al. 2012), and it seems likely that many of them are unbound. Although objects in the planetary mass range are now being found by direct observation in the infrared (Delorme et al. 2012; Beichman et al. 2013), these infrared 
surveys do not currently reach down to $\sim 1$ Jupiter mass, where the microlensing signal is seen. Unbound planets are expected from a variety of processes, including planet-planet scattering (Levison et al. 1998; Ford \& Rasio 2008; Guillochon et al. 2011), star-planet scattering (Holman \& Wiegert 1999; Musielak et al. 2005; Doolin \& Blundell 2011; Malmberg et al. 2011; Veras \& Raymond 2012; Kaib et al. 2013), and stellar mass loss and death (Veras et al. 2011; Veras \& Tout 2012; Voyatzis et al. 2013). However, most of these processes have been expected to generate fewer of these unbound or very wide orbit planets than the $1.8_{-0.8}^{+1.7}$ Jupiter-mass planets per main sequence star found by microlensing (Sumi et al. 2011).

The evidence for this isolated planet population is statistical, and is based on the distribution of the Einstein radius crossing times, $t_{E}$, for microlensing events seen toward the Galactic bulge. However, these $t_{E}$ values depend not only on the masses of the gravitational lenses, but also on the lens distances, $D_{L}$, and their relative proper motions, $\mu_{\text {rel }}$, with respect to the source stars. For a subset of events, it is possible to obtain more information. If the source star and lens object angular separation becomes very much smaller than the angular Einstein radius, $\theta_{E}$, then finite source effects may allow the measurement of the angular Einstein radius (Alcock et al. 1997). In extreme cases, terrestrial microlensing parallax effects can be measured, which will enable a direct measurement of the lens mass (Gould et al. 2009; Yee et al. 2009) and a determination of the distance to the lens.

The relative proper motion, $\mu_{\text {rel }}$, can also be measured for binary lensing events, which often have caustic crossing features that are infinitely sharp. Most binary events with planetary mass ratios of $q \lesssim 10^{-3}$ fall into this category, and this implies that the source radius crossing time, $t_{*}$, can be determined from the light curve model. The event presented in this paper is one such event, and it is also the shortest duration event detected to date with a planetary mass ratio companion. The Einstein radius crossing time is $t_{E} \simeq 3.8$ days, and the mass ratio is $q \simeq 4.7 \times 10^{-4}$. The $t_{*}$ value from the best fit model, this gives a lens-source

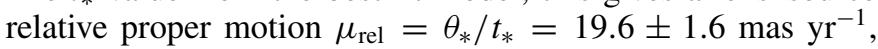
(measured in the inertial geocentric reference frame moving at the velocity of the Earth at the time of the event). Such a high relative proper motion would usually imply that the lens must be nearby, particularly since the source star proper motion is fairly tightly constrained by the OGLE data (Skowron et al. 2013). This $t_{*}$ measurement also leads to a determination of the angular Einstein radius $\theta_{E}=t_{E} \theta_{*} / t_{*}=0.205 \pm 0.016$ mas, which can be used in a mass-distance relationship. But, this $\theta_{E}$ value is quite small for a nearby lens, and this implies a total lens mass of only a few Jupiter masses if the lens system is $<1 \mathrm{kpc}$ away, as the $\mu_{\text {rel }}$ value seems to imply.

Unfortunately, the light curve for this event has one feature that complicates the interpretation. The time interval between the caustic entry and the caustic exit is quite similar to the source radius crossing time, $t_{*}$, and we find two distinct, nearly degenerate solutions, with parameters that are very similar, except that the $t_{*}$ value for the second (slow) solution is larger by a factor of 1.68 . This slow solution is disfavored by only $\Delta \chi^{2}=2.9$. The geocentric relative proper motion for this slow

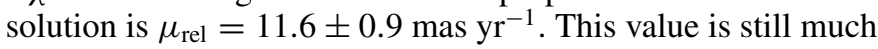
larger than the relative proper motion measured for any other planetary microlensing event, and it suggests a nearby lens. However, as we shall see below in Section 6, this preference for nearby lenses is not enough to overcome the preference for stellar-mass lens primaries due to their larger Einstein radii. So, when the appropriate prior on the mass function is included, a stellar mass or brown dwarf host is preferred.

This paper is organized as follows. We discuss the data and its collection in Section 2, and in Section 3 we present our light curve models. Section 4 presents the calibration of the optical data and the source radius estimate, while Section 5 describes constraints on the source proper motion using OGLE data and the analysis of high resolution infrared adaptive optics (AO) observations made with the Keck-2 telescope. In Section 6, we present a Bayesian analysis, which relates the observed light curve parameters to the physical parameters of the lens system, and in Section 7, we discuss how very high cadence observations from multiple sites could resolve the ambiguities in similar events using the terrestrial parallax effect. Finally, in Section 8 , we present our conclusions.

\section{DATA AND PHOTOMETRY}

Microlensing event MOA-2011-BLG-262 [(R.A., decl.) = $\left(18^{\mathrm{h}} 00^{\mathrm{m}} 23^{\mathrm{s}} .48,-31^{\circ} 14^{\prime} 42^{\prime \prime} .93\right)$ and $(l, b)=(-0.3693$, $-3.9245)$ ] was identified by the MOA alert system (Bond et al. 2001) and announced by the MOA group at 2011 June 26 14:16 UT, based on microlensing survey data from the MOA-II telescope at Mt. John University Observatory (MJUO) in New Zealand. It was immediately recognized by the MOA, PLANET, and $\mu \mathrm{FUN}$ groups that this was a short duration, high magnification event that would be highly sensitive to planetary mass ratio companions (Griest \& Safizadeh 1998; Rhie et al. 2000). The $\mu$ FUN group sent an email alert announcing this fact. Follow-up observations were immediately begun by the PLANET Collaboration, using the $1.0 \mathrm{~m}$ telescope at Canopus Observatory in Tasmania, and by MOA using the $0.61 \mathrm{~m}$ Boller $\&$ Chivens telescope, also at MJUO. The observing cadence on the MOA-II $1.8 \mathrm{~m}$ telescope was increased from one observation every 50 minutes to one observation every 2 minutes, and then cut back to one observation every 7 minutes. These followup data were enabled by the rapid identification of the event by the MOA Alert system (Bond et al. 2001), which identified the event based on the first three observations of the night and allowed the alert to be used $\sim 50$ minutes after the third observation of the night. Because of the short duration of the event, it was unclear whether observatories in Chile would be able to get useful color information for the source star, so the MOA group obtained both $V$ - and $I$-band photometry from the $0.61 \mathrm{~m}$ Boller $\&$ Chivens telescope.

At about the time that these follow-up observations began, the leading limb of the source star crossed a caustic due to a planetary mass ratio companion to the primary lens object. This caustic feature was observed by the MOA-II $1.8 \mathrm{~m}$, the MJUO $0.61 \mathrm{~m}$, and the Canopus $1.0 \mathrm{~m}$ telescopes until well after the trailing limb of the source exited the caustic $\sim 1.4 \mathrm{hr}$ later. A peak magnification of $A_{\max }=75$ was reached, as shown in Figure 1. The observations from New Zealand and Tasmania continued until night ended, when the magnification had dropped to $A<30$. During the final hour of observations from New Zealand and Eastern Australia, the Robonet Collaboration was able to observe with the $2.0 \mathrm{~m}$ Faulkes South Telescope (FTS) located at the Siding Springs Observatory, but unfortunately, this was after the planetary anomaly had ended.

Observations from Chile began at about HJD $=2455739.5$, when the magnification was about $A \approx 10$. These observations were made by the OGLE group using the $1.3 \mathrm{~m}$ Warsaw University telescope and the OGLE-IV camera at the Las Campanas Observatory and the $\mu$ FUN group using the $1.3 \mathrm{~m}$ SMARTS 

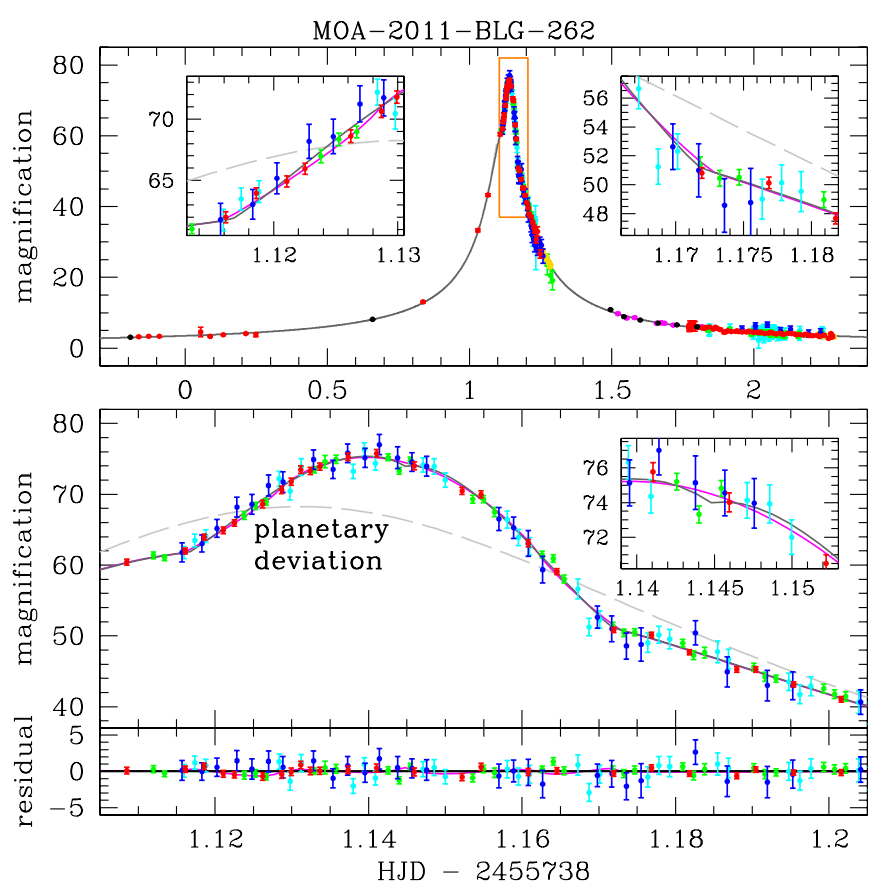

Figure 1. Light curve of event MOA-2011-BLG-262 with data from the MOA $1.8 \mathrm{~m}$ (red), Mt. John University Observatory $I$ and $V$-bands (green and cyan), Canopus $I$-band (blue), CTIO I-band (magenta), OGLE $I$-band (black), and Faulkes Telescope South (gold). The best fit model is indicated by the black curve, and the magenta curve indicates an alternative planetary model, which almost fits the light curve except for the limb crossings shown in the inserts This model has a $\chi^{2}$ larger by $\Delta \chi^{2}=2.91$ when compared to the best fit model.

telescope at the Cerro Tololo InterAmerican Observatory. Because the event was no longer at high magnification when it could be observed from Chile, the $\mu$ FUN group focused on obtaining multi-color data in the $V, I$, and $H$ passbands. While the CTIO $V$ and $I$-band data constrained the source color with less $\mathrm{S} / \mathrm{N}$ than the $V$ and $I$-band data from the MJUO $0.61 \mathrm{~m}$, the $H$-band data were unique, and proved to be necessary to normalize follow-up high resolution AO data. The CTIO $V$ and $I$ data were also used help determine the dust extinction in the vicinity of the target star. While the $\mu$ FUN observations were made as a result of the MOA alert of this event, OGLE had been observing this event previously as a part of the OGLE-IV survey. The event was discovered independently by the OGLE Early Warning System (Udalski et al. 1994) and announced as OGLE-2011-BLG-0703. Because the lens is located in a lower cadence OGLE-IV field, the alert was triggered exactly on the peak night and distributed just after the peak magnification.

We also obtained high angular resolution infrared AO followup data from the Keck-2 telscope (as discussed in Section 5, and wide-field infrared images from the InfraRed Survey Facility (IRSF) at the South African Astronomical Observatory to help calibrate the Keck data. However, data from the Vista Variables in the Via Lactea (VVV) project (Minniti et al. 2010) became available, and this was used instead of the IRSF data.

The light curve data were reduced to photometry using a number of implementations of the difference imaging photometry method (Tomaney \& Crotts 1996; Alard \& Lupton 1998). The MOA-II telescope data, consisting of 4884 observations in the MOA custom red band (roughly equivalent to Cousins $R+I$ ), and the MJUO $0.61 \mathrm{~m}$ data were reduced with the MOApipeline (Bond et al. 2001). The MJUO data consist of 143 $V$-band and $168 I$-band measurements. The $59 \mathrm{I}$-band observations from Canopus Observatory were reduced with pySIS
(Albrow et al. 2009); the 37 FTS SDSS-I-band observations were reduced with the Robonet pipeline (Bramich 2008), and the 298 OGLE-IV I-band observations were reduced with the OGLE pipeline (Udalski 2003).

As with most microlensing events, the error bars calculated by these photometry codes give only a rough estimate of the actual photometry errors. These are sufficient to find the best fit models, but error bars that give $\chi^{2} /$ dof $\approx 1$ are needed in order to estimate the errors on the physical parameters of the lens system. We have therefore followed the standard procedure of adding $0.3 \%$ in quadrature to each error estimate, and then renormalized the error bars to give $\chi^{2} /$ dof $=1$ for a preliminary planetary model (a version of the best fit model in this case). Experience with the analysis of a large number of other events indicates that the final results have no significant dependence on the details of this procedure or on which preliminary model is used to determine the error bar renormalization factors. (Virtually every planetary microlensing event that has been published has been analyzed independently with different preliminary models and error bar renormalization procedures, and the results of these different analyses are never significantly different.)

We computed the source-star limb-darkening coefficients from Kurucz's ATLAS9 stellar model atmosphere grid (Kurucz 1993a, 1993b, 1994) using the method described in Heyrovský (2007). For the photometric band of each light curve we used instrument-specific response functions obtained by combining the respective filter transmission and CCD quantum efficiency curves. We interpolated the limb-darkening coefficients for stellar effective temperature $T_{\text {eff }}=5520 \mathrm{~K}$, surface gravity $\log g=4.1$, microturbulent velocity $v_{\mathrm{t}}=2 \mathrm{~km} \mathrm{~s}^{-1}$, and solar metallicity from the values obtained for the Kuruczmodel parameter grid. These parameters were selected to be consistent with the source star properties presented in Section 4. The computed linear limb-darkening coefficients for the four light curves with sufficient caustic-crossing points are 0.5291 , $0.4861,0.6587$, and 0.4605 for MOA red, Canopus $I$, MJUO $V$, and MJUO $I$, respectively.

\section{LIGHT CURVE MODELS}

The light curve of this event, shown in Figure 1, was modeled using the image centered ray-shooting method (Bennett \& Rhie 1996; Bennett 2010). The global fit strategy used was the initial condition grid search method of Bennett (2010). However, after this grid search was run, we also did a grid search in the lens separation, $s$, in order to ensure that all degenerate solutions were recovered. (The separation, $s$ is measured in units of the Einstein Radius, $R_{E}$.) The best planetary model has a $\chi^{2}$ improvement of $\Delta \chi^{2}=1712.95$ over the best single lens model. We find four distinct $\chi^{2}$ minima, and the parameters of these solutions are given in Table 1. These four solutions are due to two degeneracies. The first degeneracy is the well known (Griest $\&$ Safizadeh 1998) 2-fold $s \leftrightarrow 1 / s$ degeneracy that occurs for most, but not all (Gaudi et al. 2008; Bennett et al. 2010; Miyake et al. 2011), planets found in high magnification events. The two best fit models, labeled "fast" in Table 1 have nearly identical parameters, except that the best model has $s=0.9578$, and its $s>1$ counterpart has $s=1.0605$ and a $\chi^{2}$ that is larger by $\Delta \chi^{2}=0.64$. These values of $s$ do not precisely correspond a $s \leftrightarrow 1 / s$ degeneracy since $1 / 0.9578=1.0441 \neq 1.0605$. This is because the shape of the central caustic is perturbed by the proximity of the planetary caustic for $s \approx 1$, and in this case, at $s=0.9578$, the planetary and central caustics have merged to form a so-called resonant caustic, as shown in Figure 2. 
Table 1

Model Parameters

\begin{tabular}{lcccccc}
\hline \hline Parameter & \multirow{2}{*}{ Units } & \multicolumn{2}{c}{ Fast } & & \multicolumn{2}{c}{ Slow } \\
\cline { 3 - 4 } \cline { 6 - 7 } & & $s<1$ & $s>1$ & & $s<1$ & $s>1$ \\
\hline$t_{E}$ & days & $3.827(13)$ & $3.846(13)$ & & $3.858(13)$ & $3.855(13)$ \\
$t_{0}$ & HJD -2455700 & $39.1312(3)$ & $39.1311(3)$ & & $39.1309(3)$ & $39.1310(3)$ \\
$u_{\text {min }}$ & & $0.01465(57)$ & $0.01451(57)$ & & $0.01470(58)$ & $0.01463(57)$ \\
$s$ & & $0.9578(11)$ & $1.0605(11)$ & & $0.9263(32)$ & $1.0966(38)$ \\
$\theta$ & radians & $1.8096(59)$ & $1.8071(59)$ & & $1.8109(60)$ & $1.8115(60)$ \\
$\epsilon$ & $10^{-4}$ & $4.66(24)$ & $4.67(24)$ & & $4.39(25)$ & $4.43(25)$ \\
$t_{*}$ & days & $0.01316(25)$ & $0.01315(25)$ & & $0.02217(27)$ & $0.02221(27)$ \\
$I_{s}$ & & $19.929(41)$ & $19.935(41)$ & & $19.937(41)$ & $19.937(41)$ \\
$V_{s}$ & & $21.888(41)$ & $21.894(41)$ & & $21.898(41)$ & $21.897(41)$ \\
fit $\chi^{2}$ & & 5757.94 & 5758.58 & & 5760.85 & 5763.82 \\
\hline
\end{tabular}

Note. The numbers in parentheses indicate the $1 \sigma$ uncertainties.

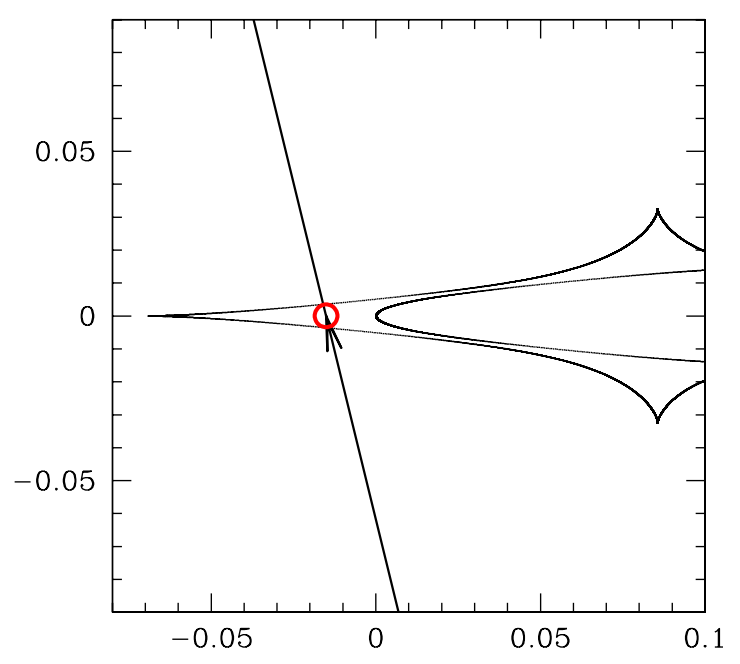

Figure 2. Caustic geometry for the best fit MOA-2011-BLG-262 "fast" model. The red circle indicates the source star size.

The other degeneracy is more unusual, and it occurs because the caustic entrance-to-exit time interval is similar to the source radius crossing time, $t_{*}$. The time interval between the caustic entry and the caustic exit is $\sim 1.4 \mathrm{hr}$, and it may be fit by models in which the sum of the source diameter crossing time and the caustic entry-exit interval is $\sim 1.4 \mathrm{hr}$. More typically, the source radius crossing time is much smaller than the caustic entranceto-exit interval (Bond et al. 2004; Miyake et al. 2011; Bachelet et al. 2012) or else the caustic is much narrower than the source (Beaulieu et al. 2006; Dong et al. 2009; Janczak et al. 2010). There are a variety of local $\chi^{2}$ minima with different caustic widths and different $t_{*}$ values, such that the caustic entrance-toexit time interval is the same. We find two distinct solutions for both the $s<1$ and $s>1$ cases with $\chi^{2}$ values within $\Delta \chi^{2}<40$ of the best fit model. The light curve features that distinguish these light curves are subtle and not easily resolved, particularly in the present case with a caustic entrance-to-exit time interval of only $\sim 1.4 \mathrm{hr}$. This is reflected in the $\chi^{2}$ difference of only $\Delta \chi^{2}=2.91$ for the second best models.

The alternate, "slow," models have $s$ values that are further from unity than the fast model, but as Table 1 indicates, the other parameters, except for $t_{*}$, are quite similar to those of the fast models. In particular, the best fit source brightnesses and color differ by $<0.01 \mathrm{mag}$ for the fast and slow models. As discussed in Section 4, this implies that the angular sources star radius, $\theta_{*}$, values will be nearly identical for these two models. Thus, the main physical difference in the two models is in the

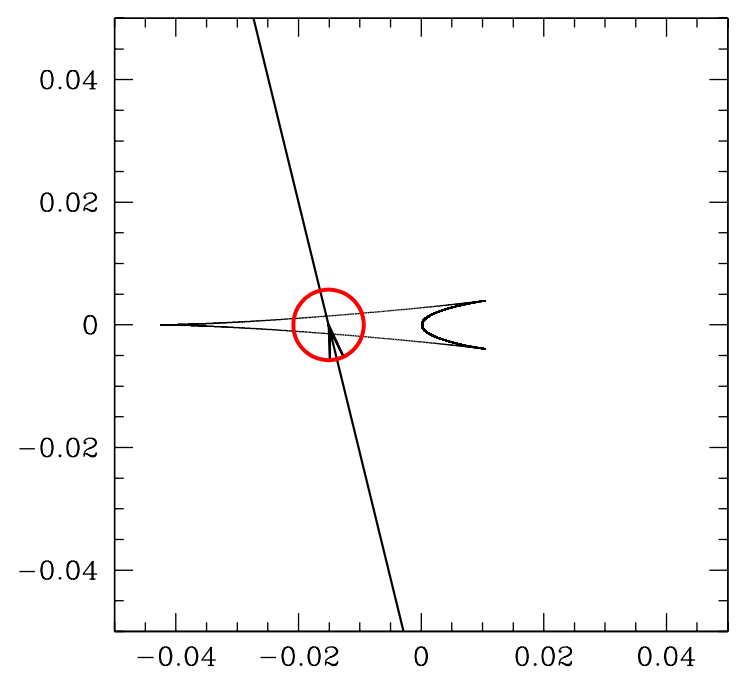

Figure 3. Caustic geometry for the best fit MOA-2011-BLG-262 "slow" model. The red circle indicates the source star size.

(geocentric) relative proper motion, $\mu_{\text {rel }}=\theta_{*} / t_{*}$. The source radius crossing times are $t_{*}=0.01316$ and $t_{*}=0.02217$ for the $s<1$ fast and slow models, respectively. In Section 4, we

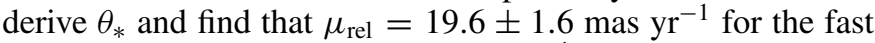

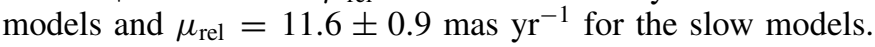
(This is the reason for the "fast" and "slow" designations.) The caustic configuration for the $s<1$ "slow" model is given in Figure 3.

Figure 1 shows the event light curve with both of the best fit $s<1$ models. The black curve is the best fit fast, $s<1$ model, which is the overall best fit, and the magenta curve is the best fit slow model (also with $s<1$ ), which is disfavored by $\Delta \chi^{2}=2.91$. The parameters of these models are given in the third and fifth columns of Table 1 . These models are difficult to distinguish, but they do differ slightly at the caustic entry and exit, as well as at the midpoint of the caustic feature where the leading limb of the source begins to exit the caustic just as the trailing limb enters for the fast model. This gives an abrupt change in slope, although the amplitude of the change is small. These three regions are highlighted in the three insets in Figure 1. The MJUO $0.61 \mathrm{~m}$ and Canopus data are not precise enough to clearly distinguish these models. The MOA-2 $1.8 \mathrm{~m}$ telescope data might be precise enough to distinguish these models if the density of observations were much higher (say one observation every minute), but with the actual observing cadence of one observation every $\sim 5$ minutes, these two models cannot be definitively distinguished. 


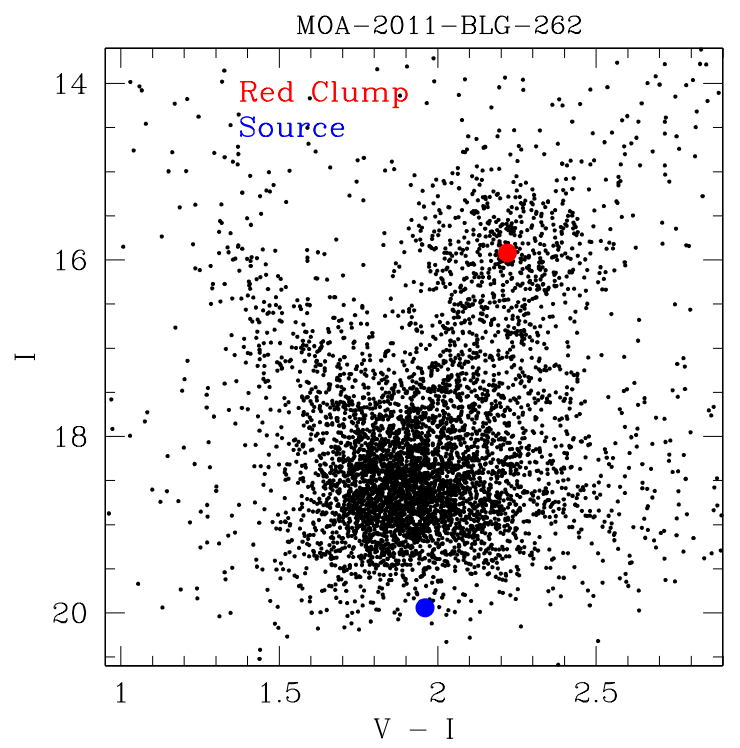

Figure 4. Color-magnitude diagram (CMD) of the stars in the CTIO images, calibrated to the OGLE-III catalog within 135" of MOA-2011-BLG-262. The red spot indicates red clump giant centroid, and the blue spot indicates the source magnitude and color.

\section{CALIBRATION AND SOURCE RADIUS}

In order to measure the angular Einstein radius, $\theta_{E}=\theta_{*} t_{E} / t_{*}$, we must determine $\theta_{*}$ from the dereddened brightness and color of the source star (Kervella \& Fouqué 2008). For most microlensing events colors are obtained from $V$ - and $I$-band measurements from either OGLE or CTIO, but this event was so short that it could only be observed at high magnification from New Zealand or Australia. Therefore, we obtained $V$ and $I$-band photometry from the MJUO $0.61 \mathrm{~m} \mathrm{B \& C}$ telescope, as shown in Figure 1. These data were calibrated using stars in the OGLE-III catalog (Szymański et al. 2011) within 2 arc minutes of the source star. However, this target is located near CCD edge in the OGLE-III reference frame, and the OGLE-III catalog does not report both $V$ and $I$ magnitudes for stars within 199 pixels (or $51^{\prime \prime} .7$ ) of the field edge, including the immediate vicinity of the source star. This means that an estimate of the extinction in the foreground of the source would be especially susceptible to errors due to differential reddening. To avoid this possibility, we use the CTIO $V$ and $I$-band photometry of this CCD to generate the color-magnitude diagram (CMD), shown in Figure 4. The CTIO data have been calibrated to the OGLE-III data base in the same way as the MJUO $0.61 \mathrm{~m} \mathrm{B \& C}$ data. The centroid of the red clump giant feature in this CMD is $I_{\mathrm{cl}}=15.92, V_{\mathrm{cl}}=18.14$, and $(V-I)_{\mathrm{cl}}=2.22$. We chose to use the CTIO data instead of the MJUO $0.61 \mathrm{~m}$ data for the CMD in order to minimize blending effects, since the CTIO images have significantly better seeing. However, the red clump centroid from the MJUO $0.61 \mathrm{~m}$ CMD is within 0.02 mag of the CTIO red clump centroid in both $(V-I)$ and $I$, so this choice does not affect our results.

Nataf et al. (2013) find that the unreddened red clump magnitude and color at this Galactic position $(l=-0.3693$, $b=-3.9245)$ are $I_{\mathrm{cl} 0}=14.47$ and $(V-I)_{\mathrm{cl} 0}=1.06$, and this implies that $A_{I}=1.45$ and $A_{V}=2.62$. This yields an extinction law with $R_{V I} \equiv A_{V} /\left(A_{V}-A_{I}\right)=2.24$ and $R_{v}(B V) \equiv A_{B} /\left(A_{B}-A_{V}\right)=2.69$, assuming the Cardelli et al. (1989) extinction law. We can then use the best fit source magnitudes listed in Table 1 to determine the dereddened source magnitudes. For the best fit (fast, $s<1$ ) model, we find
$I_{s 0}=18.359$ and $(V-I)_{s 0}=0.840$, while for the best fit slow model they are almost identical, $I_{s 0}=18.367$ and $(V-I)_{s 0}=0.841$. With these dereddened magnitudes, we can use Kervella \& Fouqué (2008) to give the angular source radius. This yields $\theta_{*}=0.778 \pm 0.059 \mu$ as for the fast $(s<1)$ model and $\theta_{*}=0.776 \pm 0.059 \mu$ as for the slow $(s<1)$ model.

Of course, the main difference between the fast and slow models is their different source radius crossing times: $t_{*}=$ 0.01316 days for the fast model, and $t_{*}=0.02217$ days for the slow model (both with $s<1$ ). These source radius crossing times give angular Einstein radii of $\theta_{E}=\theta_{*} t_{E} / t_{*}=$ $0.205 \pm 0.015$ mas and $\theta_{E}=0.122 \pm 0.009$ mas for the fast and slow models, respectively. The implied lens-source relative geocentric proper motion values are $\mu_{\text {rel }}=\theta_{*} / t_{*}=19.6 \pm$ $1.6 \mathrm{mas} \mathrm{yr}^{-1}$ for the fast model and $\mu_{\text {rel }}=11.6 \pm 0.9 \mathrm{mas} \mathrm{yr}^{-1}$ for the slow model.

\section{SOURCE STAR CONSTRAINTS}

\subsection{Source Proper Motion from OGLE Data}

With the ground-based, seeing-limited data used by microlensing surveys, it is generally not possible to measure the proper motion of stars as faint as MOA-2011-BLG-262S at $I_{s} \simeq$ 19.9. The central Galactic bulge fields, where most microlensing events are observed, are very crowded. They are so crowded that the seeing disks generally have more than one star with $I \lesssim 20$. Thus, point-spread function (PSF) fitting photometry codes, like DoPHOT (Schechter et al. 1993) or DAOPHOT (Stetson 1994) are usually unable to identify individual main sequence stars. The faint star-like images seen in these images are generally blends of multiple stars, so their apparent proper motion would actually be some average of the proper motion of the multiple stars contributing to each blend. As a result, attempts to measure proper motions with microlensing survey data (Sumi et al. 2004; Rattenbury et al. 2007) are limited to relatively bright stars. High angular resolution Hubble Space Telescope (HST; Kozłowski et al. 2006) data is needed to measure the proper motion of the faint main sequence and turn-off stars in the bulge.

The situation is significantly improved for faint microlensing event source stars with good quality light curve measurements. The microlensing signal allows both the source star position and brightness to be determined from the difference images, and microlensing model. This additional information allows its proper motion to be measured using a method developed by Skowron et al. (2013). They apply their method to MOA-2011-BLG-262S, and find its proper motion to be $\boldsymbol{\mu}_{s \text {, hel }}=$

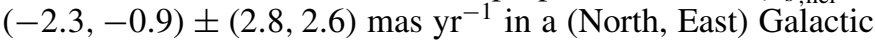
Heliocentric coordinate frame.

In order to compare with the geocentric relative proper motion values, $\mu_{\text {rel }}$, determined from the light curve models, we must convert this source proper motion value to the inertial geocentric frame that moves at the velocity that the Earth had at the light curve peak. This velocity was $\left(v_{N}, v_{E}\right)=(-0.2,29.2) \mathrm{km} \mathrm{s}^{-1}$. Assuming a source distance of $8.3 \mathrm{kpc}$, this implies a source proper motion of $\mu_{s, \text { geo }}=(-2.3,-1.7) \pm(2.8,2.6){\text { mas } \mathrm{yr}^{-1}}^{-1}$ in the geocentric frame appropriate for this event. These are the values used for the Bayesian analysis presented in Section 6.

\subsection{Keck Adaptive Optics Observations}

$J, H$, and $K$ AO images of MOA-2011-BLG-262 were taken with the NIRC2 instrument on the Keck-2 telescope on 2012 May 6, using the Laser Guide Star system. This was long after the peak of the event, so the source was no longer magnified. 


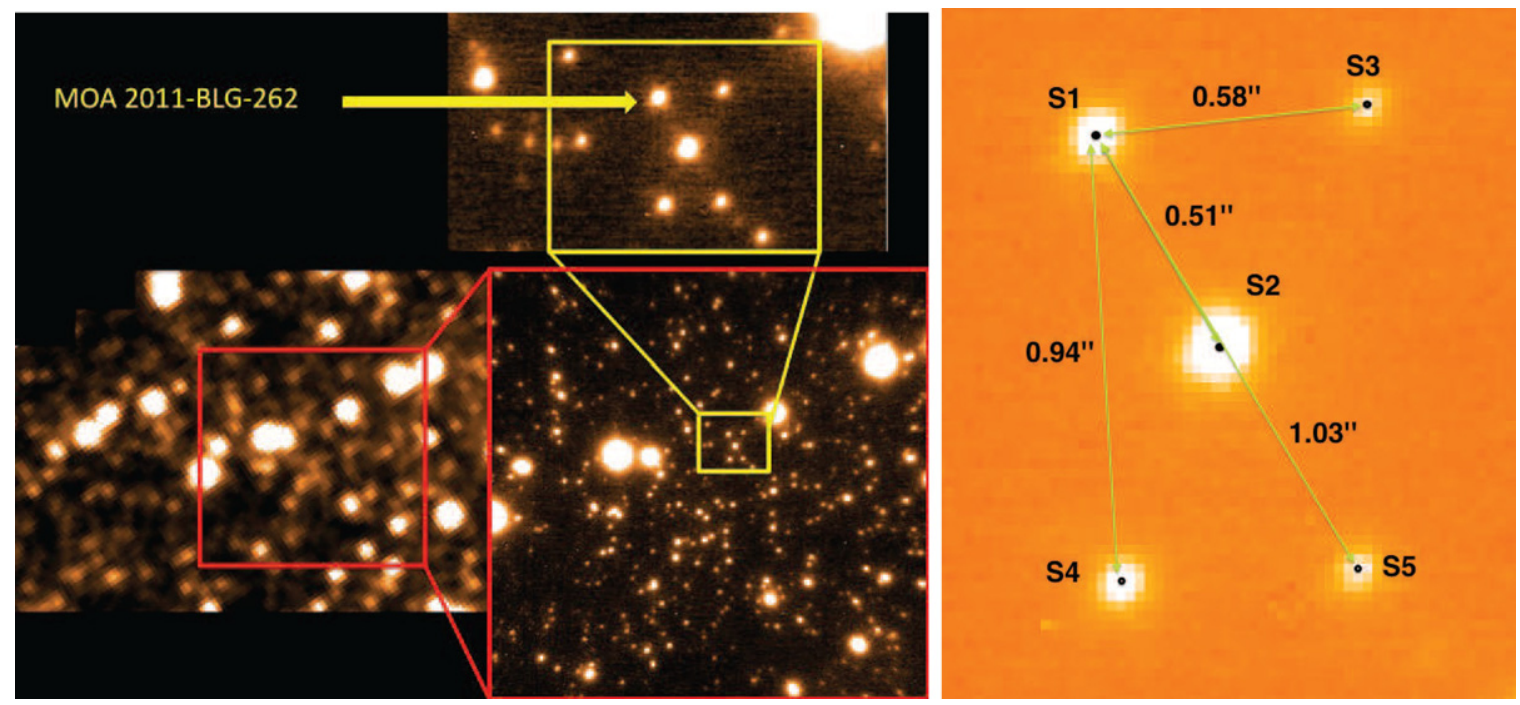

Figure 5. Left panel shows a $K$-band image from the VISTA $4 \mathrm{~m}$ telescope from the VVV survey. The field observed by the Keck-2 telescope in $K$ and a zoom of this field are shown in the middle panel. The arrow indicates the microlensing source star, and it is separated by 0.51 arcsec from its nearest neighbor. The right panel is shows a Keck-2 AO close up of the source star, S1, and four neighboring stars.

The NIRC2 medium field camera was used with a field of view of $20^{\prime \prime} \times 20^{\prime \prime}$, which has a 0.02 arcsec pixel size. (Due to $2^{\prime \prime}$ dithering, stacked images have a size of $24^{\prime \prime} \times 24^{\prime \prime}$.) Figure 5 shows the $K$-band image stack (of $20 \times 30$ s exposures) compared to a VVV image (Minniti et al. 2010) in the same band taken by the VISTA $4 \mathrm{~m}$ wide field infrared telescope at Paranal. The high resolution Keck images reveal five stars that were not resolved in the photometric light curve data used for modeling, and the star labeled S1 in Figure 5 is identified as the source star through an astrometric comparison of difference images taken near peak magnification (Skowron et al. 2013). The Keck AO images were taken less than a year after the microlensing peak, and this implies that the lens-source separation at the time of the images is $\lesssim 20$ mas. Since the seeing in the Keck images is 0.10 , a stellar lens would have its image unresolved from the source star. While this field is crowded with stars when observed in seeing-limited ground-based images, the Keck AO image appears relatively uncrowded, with the vast majority of the stars well isolated. In the following, we consider S1 as the source+lens position. A hypothetical unrelated star would be unresolved from, and add flux to the measured S1 flux, if its separation from the target is $<200$ mas.

The $J, H$, and $K$ Keck magnitudes have been measured with PSF photometry and calibrated to the Two Micron All Sky Survey (2MASS; Carpenter 2001) ${ }^{55}$ system using the JHK images from the VVV survey, as an intermediary between 2MASS and Keck. The calibrated magnitudes of star S1, which would include both the source and the lens are

$$
(J, H, K)_{\mathrm{S} 1}=(18.64,18.15,18.15) \pm(0.10,0.07,0.10)
$$

The five stars S1-S5 are not resolved in the VVV images, but stars S4 and S5 are far enough away that they are more likely to contribute to the background than the detected source in the VVV image. The detected flux in $H$ band at the lens+source position is $H_{\mathrm{S}, \mathrm{VVV}}=17.03 \pm 0.12$, which is nearly the same to the Keck measurement of the two brightest stars S1 and S2, $H_{\mathrm{S} 1+\mathrm{S} 2, \mathrm{Keck}}=16.95 \pm 0.10$.

\footnotetext{
55 Improved calibrations are available at http://www.ipac.caltech.edu/2mass/releases/allsky/doc/sec6_4b.html.
}

\subsection{Lens Flux Upper Limits from Keck Observations}

We have $H$-band data taken with the CTIO 1.3 m SMARTS during the microlensing event that allow us to determine the magnitude of the source in this passband. The best fit source magnitudes are $H_{S \text {, fast }}=18.220 \pm 0.040$ and $H_{S \text {, slow }}=$ $18.226 \pm 0.040$. These are to be subtracted from the total S1 target flux, which must include both the source and the lens system, $H_{\mathrm{S} 1}=18.15 \pm 0.07$. These measurements differ by less than $1 \sigma$, there is no significant detection of flux from the lens. But they do allow a lens flux of $H_{L \text {,fast }} \leqslant 21.16 \pm 1.10$ and $H_{L \text {,slow }} \leqslant 21.07 \pm 1.00$, where we use an inequality because of the possibility of additional flux from an unrelated star. There error bars on these source brightnesses were determined using a linear approximation, which is actually not valid, as the uncertainties do not follow Gaussian statistics (even approximately) when expressed in magnitudes. (The error bars are valid $1 \sigma$ uncertainties when converted into flux units using a linear approximation, however.) The $2 \sigma$ upper limits on the lens brightness for the fast and slow models are $H_{L \text {,fast }}>19.85$ and $H_{L, \text { slow }}>19.83$.

The lens magnitude estimates and limits can be expressed in terms of the absolute magnitude as $m_{\mathrm{H}}=H_{L}-A_{H}-$ $5 \log \left(D_{L} / 10 \mathrm{pc}\right)$, where $A_{H}$ is the extinction. From (Gonzalez et al. 2011), we obtain an $H$-band total extinction of $A_{H, \mathrm{Car}}=$ $0.39 \pm 0.11$ (Cardelli et al. 1989) or $A_{H, \text { Nish }}=0.315 \pm 0.09$ (Nishiyama et al. 2009). Assuming a linear expression for the extinction along the line of sight, we show the upper limits of the lens flux in $H$ band in Figure 6. These plots assume a fixed source distance of $D_{S}=8.3 \mathrm{kpc}$. The left and right panels indicate the relations for the fast and slow models. The magnitude distance relations for the two extinction laws are given by the nearly overlapping black dashed curves in each plot, and the gray dashed lines and shading indicate the extent of the $2 \sigma$ allowed region of parameter space. As a reference, we draw in red dashed lines the ranges for super Jupiters and brown dwarfs. We have also included isochrones for main sequence stars (An et al. 2007) in the upper right of each plot. We take the oldest population from An et al. (2007) of $4 \mathrm{Myr}$ and the following range for metallicity: $0.0 \leqslant\left[\mathrm{~F}_{\mathrm{e}} / \mathrm{H}\right] \leqslant+0.3\left(\left[\mathrm{~F}_{\mathrm{e}} / \mathrm{H}\right]=0.0\right.$ (dark blue), $\left[\mathrm{F}_{\mathrm{e}} / \mathrm{H}\right]=0.2$ (green), and, $\left.\left[\mathrm{F}_{\mathrm{e}} / \mathrm{H}\right]=0.3(\mathrm{red})\right)$. The allowed ranges for stellar 

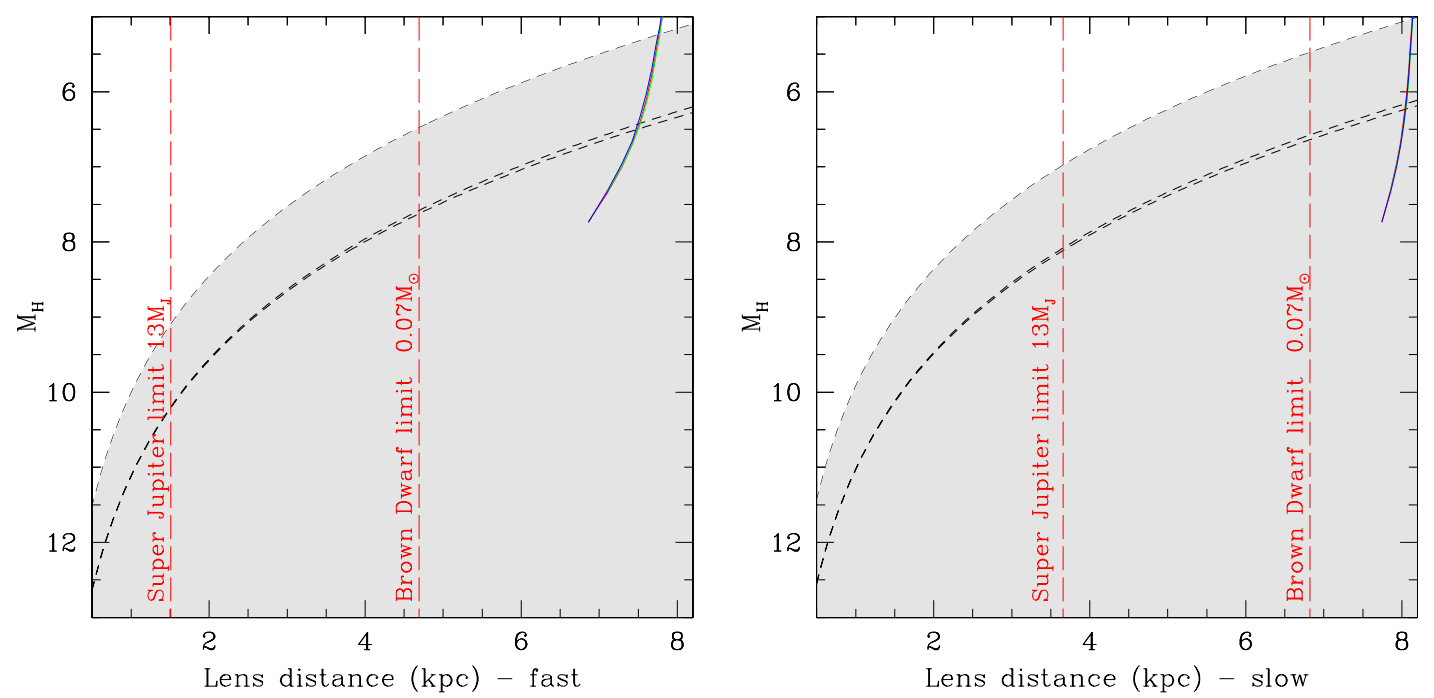

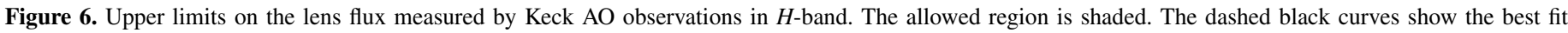

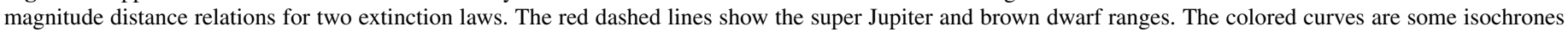
for main sequence stars from An et al. (2007).

(A color version of this figure is available in the online journal.)

hosts are on the lower part of these isochrones, below the Keck curves. The crossing points between the distance-magnitude and isochrone curves are the following for the two different models $D_{L}=7 \mathrm{kpc}$, and $D_{L}=7.7 \mathrm{kpc}$ for the fast and slow models, respectively. These imply lens host masses of $M_{\text {host }}=0.36 M_{\odot}$ and $M_{\text {host }}=0.41 M_{\odot}$, for the fast and slow models, respectively. The implied transverse velocities with respect to the source, which is known to be moving relatively slowly (Skowron et al. 2013) are quite large. For the fast model, this velocity is $V_{\text {rel }}=677 \mathrm{~km} \mathrm{~s}^{-1}$, which is likely above the escape velocity, while for the slow model, it is $V_{\text {rel }}=442$, which is very high for a bulge star.

If the fast model is correct, then as we will see below in Section 6, a host mass of only a few Jupiter masses is favored (with our assumed mass function prior). In this case it is sensible to ask the question of whether there is a host star with a separation too wide to be found by microlensing (Quanz et al. 2012). The distance to the planet+moon system favored by the fast model is $D_{L} \sim 500 \mathrm{pc}$. Since a star at the bottom on the main sequence has an absolute $H$-band magnitude of $M_{H} \approx 10.0$, its brightness at $500 \mathrm{pc}$ would be $H<18.8$ even if virtually all the dust extinction is in the foreground. Thus, we can rule such a host star blended with the lens, and the only possibility for a host star would be if it was one of the resolved stars in the Keck images. However, all the nearby stars have $(J-K) \lesssim 1$, which excludes them as possible host stars because if they were at $\sim 500 \mathrm{pc}$, they would have to be very low-mass, very red stars.

\section{BAYESIAN ANALYSIS}

Because we are unable to measure a microlensing parallax signal for this event, we have only two light curve parameters, $t_{E}$ and $\theta_{*}$ that can be used to constrain the lens mass, $M_{L}$, distance, $D_{L}$, and lens-source relative transverse velocity, $v_{\perp}$. With 2 parameters to constrain 3 unknowns, we have a one-parameter family of solutions, or a mass-distance relation,

$$
\begin{aligned}
M_{L} & =\frac{c^{2}}{4 G} \theta_{E}^{2} \frac{D_{S} D_{L}}{D_{S}-D_{L}}=\frac{c^{2}}{4 G} \theta_{E}^{2} \frac{\mathrm{AU}}{\pi_{\mathrm{rel}}} \\
& =0.9823 M_{\odot}\left(\frac{\theta_{E}}{1 \mathrm{mas}}\right)^{2}\left(\frac{x}{1-x}\right)\left(\frac{D_{S}}{8 \mathrm{kpc}}\right),
\end{aligned}
$$

where $x=D_{L} / D_{S}$ and $\theta_{E}=\theta_{*} t_{E} / t_{*}$, as discussed in Section 4. The lens-source relative parallax is given by $\pi_{\text {rel }}=\operatorname{AU}\left(D_{S}^{-1}-D_{L}^{-1}\right)$.

The relative proper motion, $\mu_{\text {rel }}$, provides a strong constraint on the properties of the lens system. While the Galactic disk moves at about the same velocity as the Sun, due to the flat Galactic rotation curve, the bulge has an average velocity of zero. So, the average proper motion of a source in the bulge is just given by the Galactic rotation speed divided by the distance

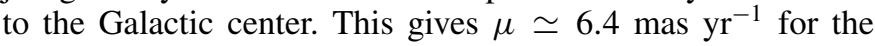
mean proper motion of a bulge star. A one-dimensional velocity dispersion of $\sim 100 \mathrm{~km} \mathrm{~s}^{-1}$ at the distance of the Galactic center gives a one-dimensional proper motion dispersion of

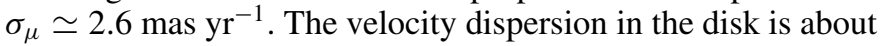
three times smaller than in the bulge, but the proper motion is inversely proportional to the distance, so the $\sigma_{\mu}$ values will be similar for a lens one third of the way to the Galactic center, at $D_{L} \simeq 2.7 \mathrm{kpc}$.

We can use these numbers to estimate the expected relative proper motion values for bulge and disk lenses. If the lens, as well as the source, is in the bulge, then the expected $\mu_{\text {rel }}$ is given by the quadrature sum of the one-dimensional dispersions in the Galactic $l$ and $b$ directions (Kozłowski et al. 2006) for

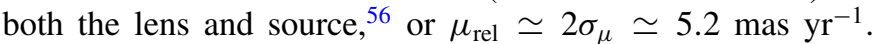
For a disk lens at $D_{L} \simeq 2.7 \mathrm{kpc}$, the average proper motion dispersion will be about the same, but there will also be the

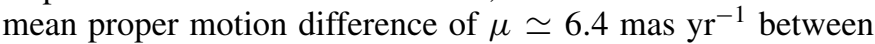
the disk and bulge. So, the average relative proper motion will be the quadrature sum of the mean proper motion and the dispersion, which yields a typical lens-source relative proper

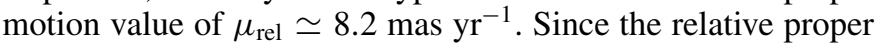
motion for both our fast and slow solutions is larger than this, the $\mu_{\text {rel }}$ values for both solutions favor a disk lens at $D_{L}<2.7 \mathrm{kpc}$. However, the degree by which a bulge lens is disfavored is quite different. For the fast solution, the measured

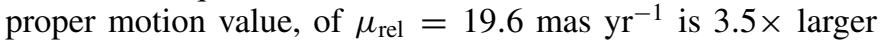

\footnotetext{
56 To get this result, we count events by the probability that an event is in progress at a given time, rather than by the event rate. This accounts for the fact that the detection efficiency for a planetary microlensing signal is approximate proportional to the event duration.
} 
than the dispersion in the proper motion difference between two bulge stars, $\mu \simeq 5.6$ mas $\mathrm{yr}^{-1}$. For the slow solution $\mu_{\text {rel }}=11.6 \mathrm{mas} \mathrm{yr}^{-1}=2.1 \times$ the bulge-bulge $\mu_{\text {rel }}$ dispersion. The Gaussian probabilities of a bulge-bulge relative proper motion outlier with $\mu_{\text {rel }}$ given by the values for our fast and slow solutions are 0.0022 and 0.11 , respectively. So, the fast solution very strongly favors a nearby lens, while the slow solution appears to only slightly disfavor a bulge lens system. (In principle, the OGLE measurement of source proper motion could change this argument, but the error bars on the source proper motion are nearly equal to the measured bulge proper motion dispersion, so the OGLE measurement has little effect at this level of analysis.)

Another important prior assumption for the Bayesian analysis is the mass function for the host mass. The only study that has looked at the mass function for the full range of masses from stellar down to planetary masses is that of Sumi et al. (2011). This study did not have much leverage on the mass function below about a Jupiter mass, so most of the models consider only a delta-function mass function for the planetarymass part of the mass function. A much better choice for this analysis is the power-law planetary mass function presented in the Supplementary Information (SI) section of Sumi et al. (2011). This is shown in Figure S11 of that paper, and the parameters of this power law model are given Table S3. This model includes stellar remnants and has a broken power law of the form $d N / d \log M=M^{1-\alpha}$, with $\alpha=2.0$ for $0.70 \leqslant M / M_{\odot} \leqslant 1.00, \alpha=1.3$ for $0.08 \leqslant M / M_{\odot} \leqslant 0.70$, $\alpha=0.49$ for $0.01 \leqslant M / M_{\odot} \leqslant 0.08$, and $\alpha=1.3$ for $10^{-5} \leqslant M / M_{\odot} \leqslant 0.01$. For $\alpha=1$, we have an equal number of objects per logarithmic mass interval, and this mass function averages close to $\alpha=1$ over the interval from low-mass stars to planetary mass objects. The ratios of main sequence stars to brown dwarfs to planets are 1:0.73:5.5 with this mass function, but most of the planetary mass objects have masses much less than that of Jupiter, because the planetary part of the mass function extends over three decades in mass. If we restrict ourselves to planetary mass objects with masses of the order of a Jupiter mass, then the numbers of main sequence stars, brown dwarfs, and planets are similar with this mass function.

While our assumed mass function implies that the densities of potential stellar, brown dwarf, and planetary mass are similar, this does not mean that the lensing probabilities are similar for these three populations. This is because the Einstein ring radius, $R_{E}=\sqrt{\left(4 G M / c^{2}\right) D_{L}\left(D_{S}-D_{L}\right) / D_{S}}$, is proportional to $\sqrt{M}$. Since the typical star has $\sim 400 \times$ Jupiter's mass, the lensing rate due to stars is enhanced by a factor of $\sim 20$ with respect to lensing by planetary mass objects. The star density in the Galactic bulge is a factor of $\sim 5$ larger than the local density in the disk. All told, this implies a factor of $\sim 100$ preference for stellar lenses over planetary mass lenses, so we should expect that the slow solution will not favor planetary mass lenses.

We use a Galactic model including a barred bulge, a spheroid, and thin and thick disks, using the functional forms for the stellar densities from Robin et al. (2003), but we use truncated Gaussians for the velocity distributions. We do not allow stars with velocities that exceed the assumed escape velocity cutoff of $550 \mathrm{~km} \mathrm{~s}^{-1}$ in the thin and thick disks, as well as in the spheroid. The assumed bulge escape velocity is $600 \mathrm{~km} \mathrm{~s}^{-1}$. Because of the high relative proper motion implied by the models for this event, the results of the Bayesian analysis may depend on the assumed velocity distributions of the Galactic model, so we have normalized the bulge velocity dispersions to the HST proper motion measurements of Kozłowski et al. (2006). Using a bar rotation velocity of $50 \mathrm{~km} \mathrm{~s}^{-1} \mathrm{kpc}^{-1}$, we can match the proper motion of the five Kozłowski et al. (2006) fields within $<1.5$ of MOA-2011-BLG-262 with velocity dispersions of $103.8 \mathrm{~km} \mathrm{~s}^{-1}$ and $96.4 \mathrm{~km} \mathrm{~s}^{-1}$ in the Galactic longitude and latitude directions, respectively.

The Bayesian priors discussed so far are all based on measurements of the properties of the stars, brown dwarfs and planets that are part of the Galaxy. However, we still must make one prior assumption for which we have no data to guide us. We will assume that probability of a lens primary to host a companion with the measured mass ratio at a separation of $\sim R_{E}$ is independent of the mass of the host. For stellar mass hosts, we have data that show that planetary mass ratio secondaries are common at these separations (Gould et al. 2010; Cassan et al. 2012), but for brown dwarf and planetary mass hosts, this is simply an assumption.

The Bayesian analysis is done with a collection of Markov Chains centered at the parameter space locations of the four solutions listed in Table 1. These are the fast and slow solutions for both $s<1$ and $s>1$. The Markov Chains for each local minima are weighted by the $\chi^{2}$ difference between the best fit model in each region of parameter space. There were two Markov Chains for each of these models, and each chain had about 350,000 links. The result of this Bayesian analysis is given in Figure 7, while Figures 8 and 9, respectively, give the results for the fast and slow models only.

Figure 7 shows the probability distribution of the host and planet masses, their projected separation, and the distance to the lens system, under the assumption that planetary and stellar mass hosts are equally likely to host a moon or planet with the observed mass ratio. There are two peaks in each distribution-one centered on planetary mass hosts at $D_{L}=$ $0.64_{-0.21}^{+0.32} \mathrm{kpc}$, and the other centered on stars just above the hydrogen-burning threshold in the central Galactic bulge at $D_{L}=7.0_{-1.0}^{+0.9} \mathrm{kpc}$. The probability drops to virtually zero at $D_{L} \sim 3.6 \mathrm{kpc}$, so we have two separate distributions: the nearby, planetary mass hosts and the stellar/brown dwarf hosts in the bulge. For the planetary mass distribution, the host and companion masses are $M_{\text {host }}=3.6_{-1.7}^{+2.0} M_{\text {Jup }}$ and $m_{\text {comp }}=$ $0.54_{-0.19}^{+0.30} M_{\oplus}$, respectively, and their projected separation is $a_{\perp}=0.13_{0.04}^{+0.06}$ AU. For the stellar/brown dwarf host solutions, the host and companion masses are $M_{\text {host }}=0.12_{-0.06}^{+0.19} M_{\odot}$ and $m_{\text {comp }}=18_{-10}^{+28} M_{\oplus}$, at a projected separation of $a_{\perp}=$ $0.84_{-0.14}^{+0.25} \mathrm{AU}$.

Figures 8 and 9 show that the planetary mass hosts come almost exclusively from the fast solution Markov Chains and that the slow solutions predict a stellar mass host located in the bulge.

We should also note that without the OGLE constraint on the source proper motion (Skowron et al. 2013), the Bayesian probability distribution for the fast solutions would resemble Figure 7 with the similar sized peaks for both nearby disk planetary mass hosts and bulge low-mass star hosts. The OGLE source proper motion constraint nearly rules out the possibility that the source is in the high velocity tail of the bulge velocity distribution. For the slow solution, it is still possible to recover the observed lens-source relative proper motion of $\mu_{\text {rel }}=$

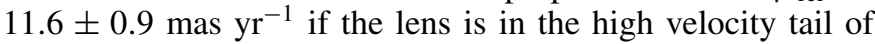
the bulge velocity distribution. The fact that stars have a much higher lensing cross section than planetary mass options enables these solutions to be favored over the nearby planetary mass 

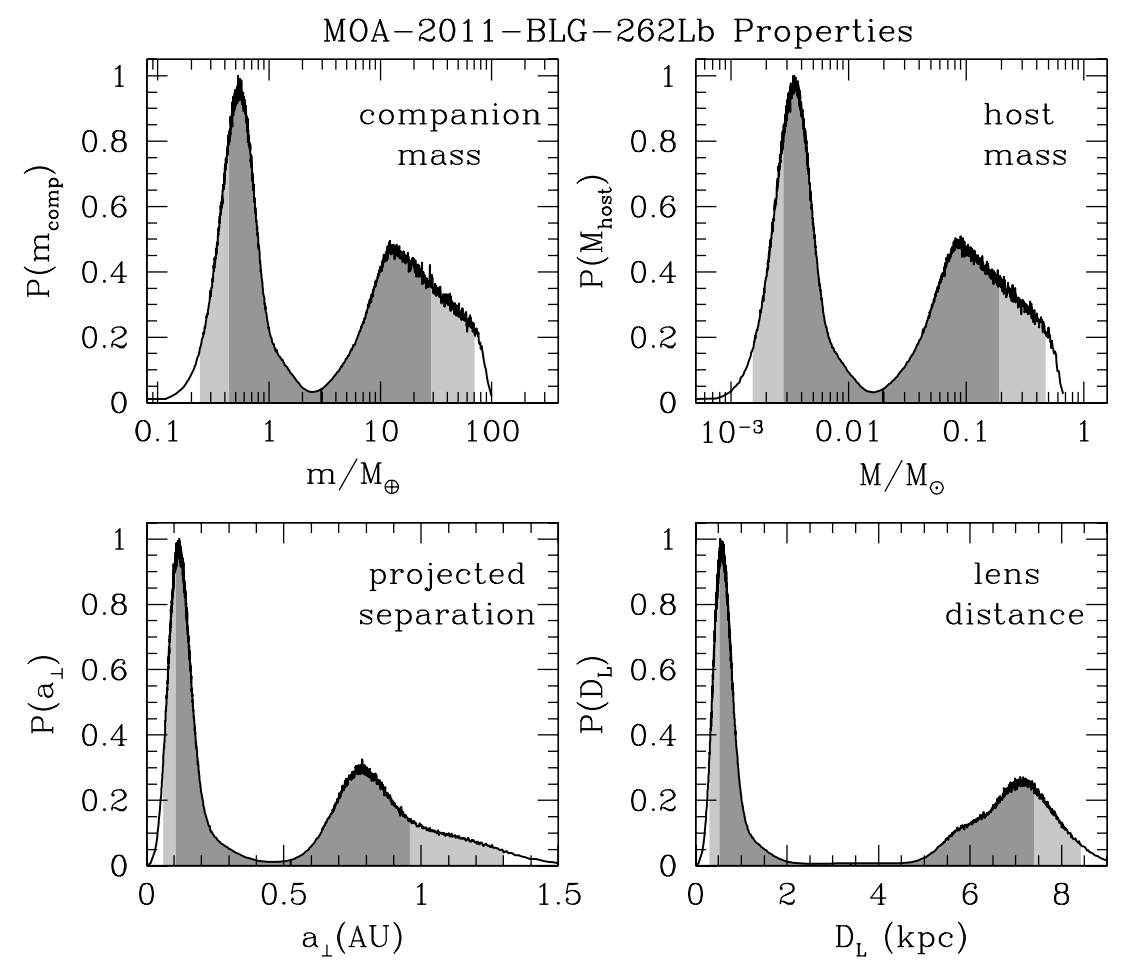

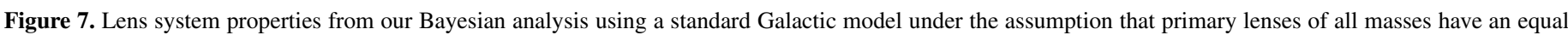

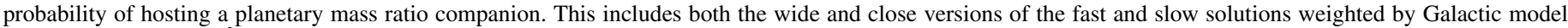
priors and the best fit $\chi^{2}$ for in each region of parameter space.
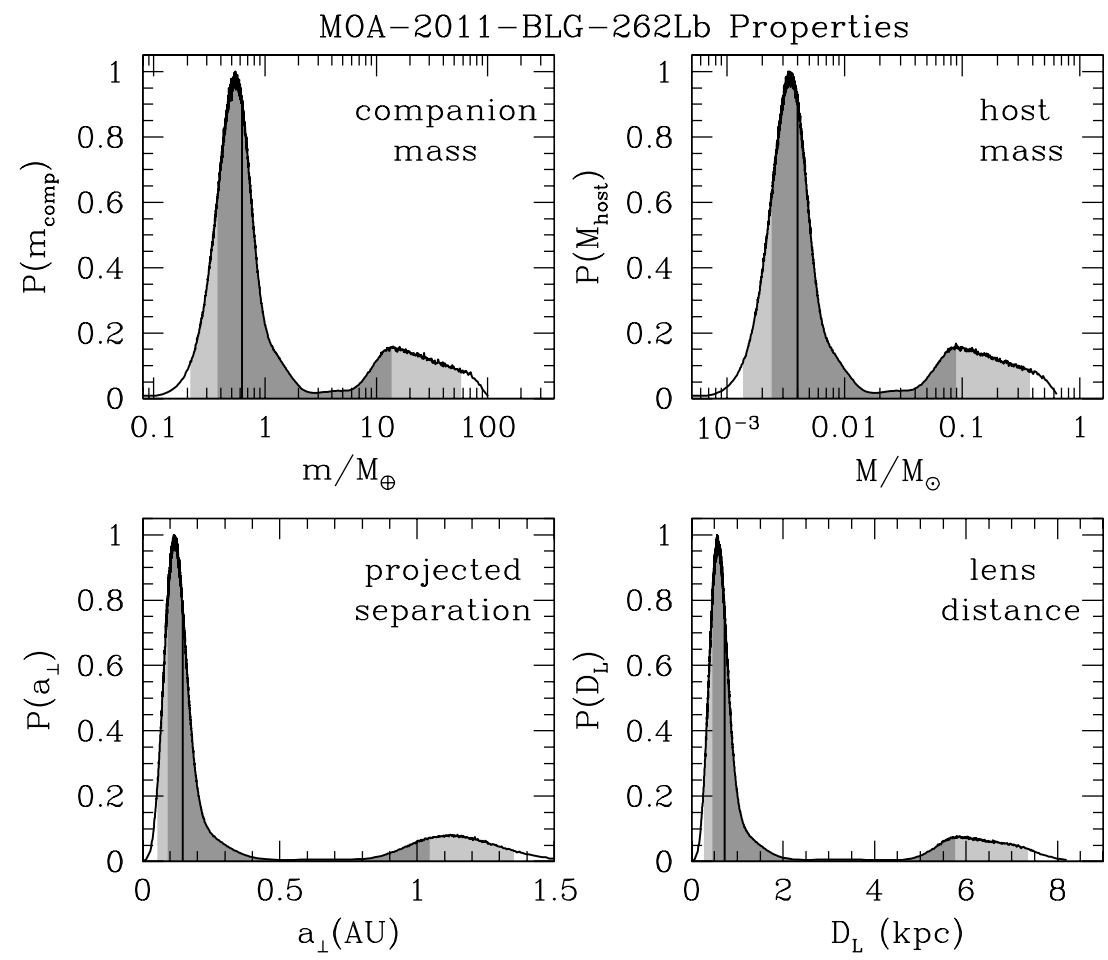

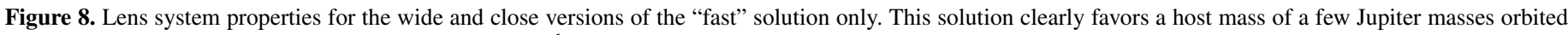
by a companion of $\sim 0.5 M_{\oplus}$. The lens system would be $\lesssim 600 \mathrm{pc}$ from the Earth.

solutions even though the $\mu_{\text {rel }}$ value itself does favor a nearby disk lens.

The situation with the fast solutions is somewhat different. The very high relative proper motions, $\mu_{\text {rel }}=19.6 \pm$

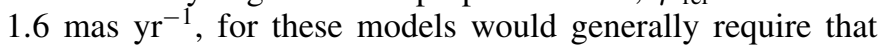
both the lens and the source belong to the high velocity tail of the bulge velocity dispersion. Since the OGLE source proper motion constraint nearly rules this out, bulge lenses are significantly disfavored with these models. So, the fast solutions, if one of them is correct, do seem to suggest that the lens system could consist of a rogue planet of a few Jupiter masses orbited by a sub-Earth-mass moon. If the fast model was the only light curve model consistent with the light curve, then the planet+moon interpretation could be confirmed with a higher 

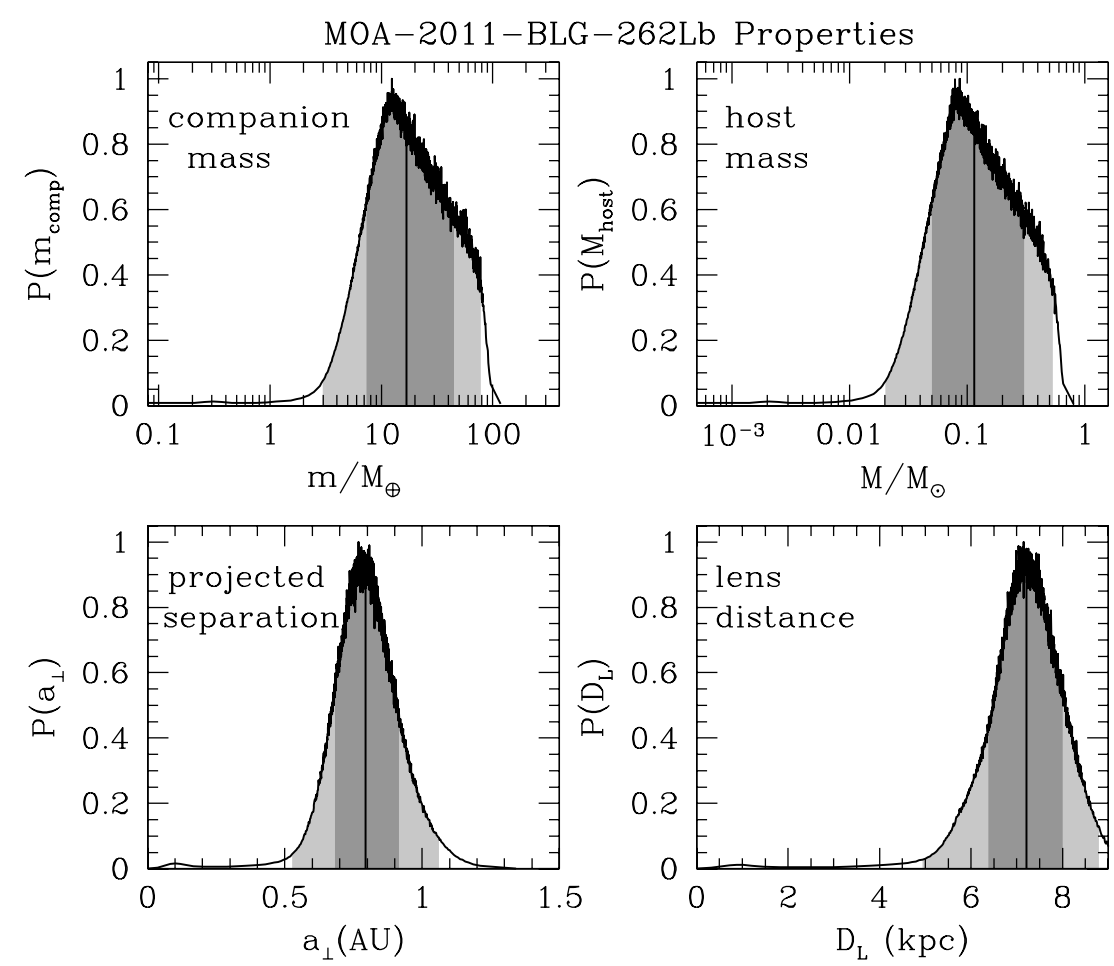

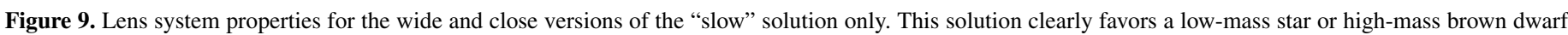

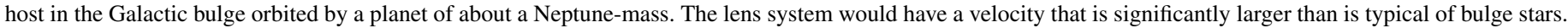

precision measurement of the source proper motion using the HST or AO observations.

\section{TERRESTRIAL PARALLAX}

The simultaneous observations of the MOA-2011-BLG-262 microlensing event from MJUO on New Zealand's South Island, and Canopus in Tasmania, Australia are potentially very useful. Although these observatories are only separated by about $2000 \mathrm{~km}$, this separation is potentially large enough so that the observed light curves would be significantly different as observed from the two observatories, due to the terrestrial parallax effect (Gould et al. 2009; Yee et al. 2009). According to the Bayesian analysis presented in Section 6, the favored solution for the fast model has a host mass of $M_{\text {host }}=$ $3.6_{-1.7}^{+2.0} M_{\text {Jup }}$ at a distance of $D_{L}=0.64_{-0.21}^{+0.32} \mathrm{kpc}$. Such a model predicts terrestrial parallax magnification differences between MJUO and Canopus of $\sim 0.5 \%$. This compares to error bars of $\sim 0.7 \%$ for the MOA-II data at the peak, so if the Canopus error bars were comparable (and preferably with a higher observing cadence), terrestrial parallax could be measured for the case that the fast planetary-mass host model is correct. Unfortunately, due to a telescope hardware issue, the images taken from Canopus during this event had unusually poor image quality, so the Canopus photometry was not precise enough for a terrestrial parallax measurement even if the fast light curve model with a nearby, planetary mass lens system was correct.

If we had photometry that allowed a terrestrial microlensing parallax measurement, then the implications of the nearly degenerate fast and slow solutions would be relatively modest. The lens masses implied by the two models would differ by only the factor of 1.7 uncertainty in $t_{*}$. In fact, the uncertainty could be much less as better photometry might be able to determine which of these models is correct. As we saw in Section 6, the Bayesian analysis that we must use without a parallax measurement results in a large uncertainty in the properties of the lens system.
Fortunately, it should be possible to definitely distinguish similar models for future events if they are observed with high cadence from multiple sites. Very high cadence observations on 1-2 m class telescopes are able to measure the light curves precisely enough to distinguish similar models (Gould et al. 2006), such as the fast and slow models for MOA-2011-BLG-262. If high cadence observations are taken from observatories separated by thousands of kilometers, then the lens system mass can be measured via the terrestrial parallax effect (Gould \& Yee 2013). Future wide-field, multi-site surveys (Park et al. 2012; Kim et al. 2013) will significantly increase the microlensing exoplanet detection rate. However, real-time planetary signal detection, such as that demonstrated by the MOA Collaboration for this event, and high cadence follow-up observations will be necessary to take full advantage of these new powerful surveys. The larger telescopes of the older microlensing follow-up groups, such as PLANET (Beaulieu et al. 2006) and $\mu$ FUN (Gould et al. 2010), can contribute some of the required high cadence follow-up observations, but the development of large robotic networks of $1 \mathrm{~m}$ class telescopes, such as the Las Cumbres Robotic Telescope Network (Brown et al. 2013), will substantially improve the rate of terrestrial microlensing parallax mass measurements. Thus, if systems resembling the planetary-mass host models for MOA-2011-BLG-262 are common, the combination of high cadence microlensing surveys, rapid realtime event detection by these surveys, and high cadence follow-up observations should enable the definitive discovery of rogue exoplanets with moons of nearly an Earth mass within a few years.

\section{SUMMARY AND CONCLUSIONS}

We have presented the analysis of microlensing event MOA2011-BLG-262, which is the shortest duration microlensing event with a planetary mass ratio of $q=4.7 \times 10^{-4}$. The best fit "fast" model implies a high lens-source relative proper of 


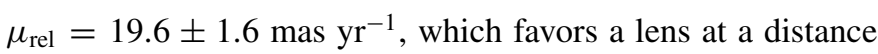
of order $D_{L} \sim 500 \mathrm{pc}$. The lens mass-distance relation (see Equation (2)) that comes from this $\mu_{\text {rel }}$ measurement implies that the lens primary for this model would have mass of 3 or 4 Jupiter masses, and the planetary mass ratio companion would be a moon with a mass of $<1 M_{\oplus}$. However, the Bayesian analysis reveals that a stellar lens host is not excluded by this fast model. Also, the source radius crossing time, $t_{*}$, is similar to the time interval between the caustic entry and exit, and this allows for another solution is a somewhat larger $t *$ value, which implies a smaller lens-source relative proper motion,

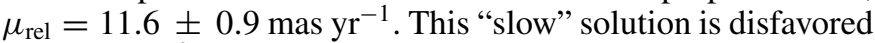
by only $\Delta \chi^{2}=2.91$, so it definitely cannot be excluded. Although the $\mu_{\text {rel }}$ for this event is also high enough to favor a nearby disk lens, it is much more compatible with a fast moving stellar lens in the bulge than the fast solution.

These relatively large relative proper motion values can largely be attributed to the motion of the lens, because the OGLE Collaboration has used a novel method to show that the source proper motion is low (Skowron et al. 2013). In order to determine the likely physical properties of the lens system, we perform a Bayesian analysis with a standard Galactic model with bulge velocities normalized to the proper motion measurements of Kozłowski et al. (2006). Since planetary mass hosts are possible for this event, we have used the power-law planetary mass model of Sumi et al. (2011; see the Supplementary Information) as our mass function prior. The only prior that is unconstrained by data is the probability that a lens primary of a given mass will host a companion lens with a mass ratio of $q=4.7 \times 10^{-4}$ at a separation of about an Einstein radius. For simplicity, we assume that this probability is independent of the primary lens mass. With such an assumption, we find two distinct types of lens systems that could explain this event, and with our assumed prior probabilities, the probability of these two solutions are roughly equal, as indicated in Figure 7.

The fast solution favors, but does not require, a planetary mass for the host, as shown in Figure 8. However, high angular resolution observations with $\mathrm{AO}$ or the $H S T$ could improve the precision of the proper motion measurement (Skowron et al. 2013), and exclude the non-planetary mass host models for the fast solution. So, if the slow solution was not viable, it would be possible to confirm the planet+moon model.

The "most likely" solution found by our Bayesian analysis for this fast solution $M_{L}=3.2 M_{\text {Jup }}$ orbited by a moon of $m_{m}=$ $0.47 M_{\oplus}$ at a three-dimensional separation of $a=0.13 \mathrm{AU}$, with the lens system at a distance of $D_{L}=0.56 \mathrm{kpc}$. The slow solution has roughly equal probability (with our prior assumptions) and implies a very different lens system. With the slow solution, the host would most likely be a star with $M_{L}=$ $0.11_{-0.06}^{+0.21} M_{\odot}$ orbited by a planet of mass $m_{p}=17_{-10}^{+28} M_{\oplus}$ at a three-dimension separation of $a=0.95_{-0.19}^{+0.53} \mathrm{AU}$ at a distance of $D_{L}=7.2 \pm 0.8 \mathrm{kpc}$, as shown in Figure 9. These parameters are quite similar to the similar to the parameters for the secondary peak for the fast solution, shown in Figure 8, except that the fast solution predicts a larger planet-star separation (both the three-dimension and projected separation). Thus, the Bayesian analysis does not directly imply a preferred solution.

The Bayesian probabilities are dependent on our prior assumption about the probability of free-floating planets to host a massive moon at a separation of $\sim 0.15 \mathrm{AU}$. However, we do know that planets exist with similar parameters to the stellar mass host favored by the slow solution (Bennett et al. 2008; Kubas et al. 2012; Furusawa et al. 2013) and consistent with the fast solution, but we do not know of any other planetary mass hosts with moons of about half an Earth-mass. There are a handful of well sampled high magnification events with $t_{E}<2$ days, which implies that they probably have planetary mass primaries. For these events, MOA-ip-10 (Sumi et al. 2011), MOA-2009BLG-450, and MOA-2010-BLG-418, we can largely exclude a companion with parameters similar to MOA-2011-BLG-262Lb. This implies that planetary mass ratio secondaries are not much more common around planetary mass hosts than around stars and brown dwarfs. So, our Bayesian prior is not likely to underestimate the probability of the planet+moon model by a significant amount. On the other hand, such systems could be extremely rare, so our Bayesian prior could greatly overestimate the probability of such a system. Or to put this another way, an apparently free-floating planet with a half Earth-mass moon would be a new class of system that was not previously known to exist. Such a new discovery would require strong evidence, so our favored model for this event is that it is a low-mass star or brown dwarf orbited by a planet of about Neptune's mass.

Improvements in the observational capabilities in the near future should allow the definitive detection of isolated planet+moon systems, like the one favored by our Bayesian analysis for the best fit (fast) model. Improved microlensing survey (Park et al. 2012) and follow-up (Brown et al. 2013) capabilities, when coupled with the rapid event identification demonstrated by the MOA group for this event, should allow the measurement of the terrestrial parallax effect (Gould et al. 2009; Yee et al. 2009), which would give a direct measurement of the lens system mass. In the somewhat more distant future, a future space-based microlensing survey (Bennett \& Rhie 2002), such as the planned WFIRST mission (Green et al. 2012; Spergel et al. 2013) or a microlensing program with the Euclid mission (Penny et al. 2013) will be even more sensitive to such events, and will enable the detection of much lower mass lens primaries. Depending on the details of the WFIRST orbit, this mission will also offer unique ways to measure the microlensing parallax (Yee 2013) and therefore determine the lens masses. Thus, although our conclusions regarding MOA-2011-BLG-262 are uncertain, future microlensing surveys will be able to determine if there is a large population of free-floating planets hosting Earth-mass moons.

D.P.B. was supported by grants NASA-NNX12AF54G, JPL-RSA 1453175 and NSF AST-1211875. This MOA project is supported by the grants JSPS18253002 and JSPS20340052. T.S. acknowledges the financial support from the JSPS, JSPS23340044, JSPS24253004. This work was partially supported by a NASA Keck PI Data Award, administered by the NASA Exoplanet Science Institute. Data presented herein were obtained at the W. M. Keck Observatory from telescope time allocated to the National Aeronautics and Space Administration through the agency's scientific partnership with the California Institute of Technology and the University of California. The Observatory was made possible by the generous financial support of the W. M. Keck Foundation. B.S.G. and A.G. were supported by NSF grant AST 110347. B.S.G., A.G., R.P.G. were supported by NASA grant NNX12AB99G. S.D. was partly supported through a Ralph E. and Doris M. Hansmann Membership at the IAS and by NSF grant AST-0807444. Work by J.C.Y. was performed in part under contract with the California Institute of Technology (Caltech) funded by NASA through the Sagan Fellowship Program. The OGLE project has received funding from the European Research Council under 
the European Community's Seventh Framework Programme (FP7/2007-2013)/ERC grant agreement No. 246678 to A.U. D.H. was supported by Czech Science Foundation grant GACR P209/10/1318. D.M.B., M.D., K.H., C.S., R.A.S., M.H. and Y.T. are supported by NPRP grant NPRP-09-476-1-78 from the Qatar National Research Fund (a member of the Qatar Foundation).

\section{REFERENCES}

Alard, C., \& Lupton, R. H. 1998, ApJ, 503, 325

Albrow, M. D., Horne, K., Bramich, D. M., et al. 2009, MNRAS, 397, 2099

Alcock, C., Allen, W. H., Allsman, R. A., et al. 1997, ApJ, 491, 436

An, D., Terndrup, D. M., Pinsonneault, M. H., et al. 2007, ApJ, 655, 233

Bachelet, E., Fouqué, P., Han, C., et al. 2012, A\&A, 547, A55

Batalha, N. M., Rowe, J. F., Bryson, S. T., et al. 2013, ApJS, 204, 24

Beaulieu, J.-P., Bennett, D. P., Fouqué, P., et al. 2006, Natur, 439, 437

Beichman, C., Gelino, C. R., Kirkpatrick, J. D., et al. 2013, ApJ, 764, 101

Bennett, D. P. 2008, in Exoplanets, ed. J. Mason (Berlin: Springer), 47

Bennett, D. P. 2010, ApJ, 716, 1408

Bennett, D. P., Bond, I. A., Udalski, A., et al. 2008, ApJ, 684, 663

Bennett, D. P., \& Rhie, S. H. 1996, ApJ, 472, 660

Bennett, D. P., \& Rhie, S. H. 2002, ApJ, 574, 985

Bennett, D. P., Rhie, S. H., Nikolaev, S., et al. 2010, ApJ, 713, 837

Bennett, D. P., Sumi, T., Bond, I. A., et al. 2012, ApJ, 757, 119

Bond, I. A., Abe, F., Dodd, R. J., et al. 2001, MNRAS, 327, 868

Bond, I. A., Udalski, A., \& Jaroszyński, M. 2004, ApJL, 606, L155

Borucki, W. J., Koch, D. G., Basri, G., et al. 2011, ApJ, 736, 19

Boss, A. P. 2006, ApJ, 643, 501

Bramich, D. M. 2008, MNRAS, 386, L77

Brown, T. M., Baliber, N., Bianco, F. B., et al. 2013, PASP, 125, 1031

Butler, R. P., Wright, J. T., Marcy, G. W., et al. 2006, ApJ, 646, 505

Cardelli, J. A., Clayton, G. C., \& Mathis, J. S. 1989, ApJ, 345, 245

Carpenter, J. M. 2001, AJ, 121, 2851

Cassan, A., Kubas, D., Beaulieu, J.-P., et al. 2012, Natur, 481, 167

Delorme, P., Gagné, J., Malo, L., et al. 2012, A\&A, 548, A26

Dong, S., Bond, I. A., Gould, A., et al. 2009, ApJ, 698, 1826

Doolin, S., \& Blundell, K. M. 2011, MNRAS, 418, 2656

Ford, E. B., \& Rasio, F. A. 2008, ApJ, 686, 621

Furusawa, Udalski, A., Sumi, T., et al. 2013, ApJ, 779, 91

Gaudi, B. S. 2012, ARA\&A, 50, 411

Gaudi, B. S., Bennett, D. P., Udalski, A., et al. 2008, Sci, 319, 927

Gonzalez, O. A., Rejkuba, M., Zoccali, M., Valenti, E., \& Minniti, D. 2011, A\&A, 534, A3

Gould, A., Dong, S., Gaudi, B. S., et al. 2010, ApJ, 720, 1073

Gould, A., \& Loeb, A. 1992, ApJ, 396, 104

Gould, A., Udalski, A., An, D., et al. 2006, ApJL, 644, L37

Gould, A., Udalski, A., Monard, B., et al. 2009, ApJL, 698, L147

Gould, A., \& Yee, J. C. 2013, ApJ, 764, 107

Green, J., Schechter, P., Baltay, C., et al. 2012, arXiv:1208.4012

Griest, K., \& Safizadeh, N. 1998, ApJ, 500, 37

Guillochon, J., Ramirez-Ruiz, E., \& Lin, D. 2011, ApJ, 732, 74

Heyrovský, D. 2007, ApJ, 656, 483

Holman, M. J., \& Wiegert, P. A. 1999, AJ, 117, 621

Ida, S., \& Lin, D. N. C. 2005, ApJ, 626, 1045
Janczak, J., Fukui, A., Dong, S., et al. 2010, ApJ, 711, 731

Johnson, J. A., Aller, K. M., Howard, A. W., \& Crepp, J. R. 2010, PASP, 122,905

Johnson, J. A., Butler, R. P., Marcy, G. W., et al. 2007, ApJ, 670, 833

Kaib, N. A., Raymond, S. N., \& Duncan, M. 2013, Natur, 493, 381

Kennedy, G. M., \& Kenyon, S. J. 2008, ApJ, 673, 502

Kennedy, G. M., Kenyon, S. J., \& Bromley, B. C. 2006, ApJL, 650, L139

Kervella, P., \& Fouqué, P. 2008, A\&A, 491, 855

Kim, D. J., Lee, C. U., Kim, S. L., \& Park, B. G. 2013, PKAS, 28, 1

Kozłowski, S., Woźniak, P. R., Mao, S., et al. 2006, MNRAS, 370, 435

Kubas, D., Beaulieu, J. P., Bennett, D. P., et al. 2012, A\&A, 540, A78

Kurucz, R. L. 1993a, Kurucz CD-ROM 16 (Cambridge, MA: SAO)

Kurucz, R. L. 1993b, Kurucz CD-ROM 17 (Cambridge, MA: SAO)

Kurucz, R. L. 1994, Kurucz CD-ROM 19 (Cambridge, MA: SAO)

Laughlin, G., Bodenheimer, P., \& Adams, F. C. 2004, ApJL, 612, L73

Lecar, M., Podolak, M., Sasselov, D., \& Chiang, E. 2006, ApJ, 640, 1115

Levison, H. F., Lissauer, J. J., \& Duncan, M. J. 1998, AJ, 116, 1998

Lissauer, J. J. 1993, ARA\&A, 31, 129

Malmberg, D., Davies, M. B., \& Heggie, D. C. 2011, MNRAS, 411, 859

Mao, S., \& Paczyński, B. 1991, ApJL, 374, L37

Mayor, M., \& Queloz, D. 2012, NewAR, 56, 19

Minniti, D., Lucas, P. W., Emerson, J. P., et al. 2010, NewA, 15, 433

Miyake, N., Sumi, T., Dong, S., et al. 2011, ApJ, 728, 120

Montet, B. T., Crepp, J. R., Johnson, J. A., Howard, A. W., \& Marcy, G. W. 2014, ApJ, 781, 28

Musielak, Z. E., Cuntz, M., Marshall, E. A., \& Stuit, T. D. 2005, A\&A, 434, 355

Nataf, D. M., Gould, A., Fouqué, P., et al. 2013, ApJ, 769, 88

Nishiyama, S., Tamura, M., Hatano, H., et al. 2009, ApJ, 696, 1407

Park, B.-G., Kim, S.-L., Lee, J. W., et al. 2012, Proc. SPIE, 8444, 844447-1

Penny, M. T., Kerins, E., Rattenbury, N., et al. 2013, MNRAS, 434, 2

Pollack, J. B., Hubickyj, O., Bodenheimer, P., et al. 1996, Icar, 124, 62

Quanz, S. P., Lafrenière, D., Meyer, M. R., Reggiani, M. M., \& Buenzli, E. 2012, A\&A, 541, A133

Rattenbury, N. J., Mao, S., Debattista, V. P., et al. 2007, MNRAS, 378, 1165

Rhie, S. H., Bennett, D. P., Becker, A. C., et al. 2000, ApJ, 533, 378

Robin, A. C., Reylé, C., Derrière, S., \& Picaud, S. 2003, A\&A, 409, 523

Schechter, P. L., Mateo, M., \& Saha, A. 1993, PASP, 105, 1342

Skowron, J., Udalski, A., Szymanski, M., et al. 2013, ApJ, submitted

Spergel, D., Gehrels, N., Breckinridge, J., et al. 2013, arXiv:1305.5422

Stetson, P. B. 1994, PASP, 106, 250

Sumi, T., Bennett, D. P., Bond, I. A., et al. 2010, ApJ, 710, 1641

Sumi, T., Kamiya, K., Bennett, D. P., et al. 2011, Natur, 473, 349

Sumi, T., Wu, X., Udalski, A., et al. 2004, MNRAS, 348, 1439

Szymański, M. K., Udalski, A., Soszyński, I., et al. 2011, AcA, 61,83

Thommes, E. W., Matsumura, S., \& Rasio, F. A. 2008, Sci, 321, 814

Tomaney, A. B., \& Crotts, A. P. S. 1996, AJ, 112, 2872

Udalski, A. 2003, AcA, 53, 291

Udalski, A., Szymański, M., Kałużny, J., et al. 1994, AcA, 44, 227

Veras, D., \& Raymond, S. N. 2012, MNRAS, 421, L117

Veras, D., \& Tout, C. A. 2012, MNRAS, 422, 1648

Veras, D., Wyatt, M. C., Mustill, A. J., Bonsor, A., \& Eldridge, J. J. 2011, MNRAS, 417, 2104

Voyatzis, G., Hadjidemetriou, J. D., Veras, D., \& Varvoglis, H. 2013, MNRAS, 430, 3383

Yee, J. C. 2013, ApJL, 770, L31

Yee, J. C., Udalski, A., Sumi, T., et al. 2009, ApJ, 703, 2082 